\title{
Astrometric Microlensing with the GAIA satellite
}

\author{
V.A. Belokurov \& N.W. Evans \\ Theoretical Physics, 1 Keble Rd, Oxford, OX1 3NP
}

6 September 2018

\begin{abstract}
GAIA is the "super-Hipparcos" survey satellite selected as a Cornerstone 6 mission by the European Space Agency. GAIA can measure microlensing by the brightening of source stars. For the broad $\mathrm{G}$ band photometer, the all-sky source-averaged photometric optical depth is $\sim 10^{-7}$. There are $\sim 1300$ photometric microlensing events for which GAIA will measure at least one datapoint on the amplified lightcurve. GAIA can also measure microlensing by the small excursions of the light centroid that occur during events. The all-sky source-averaged astrometric microlensing optical depth is $\sim 2.5 \times 10^{-5}$. Some $\sim 25000$ sources will have a significant variation of the centroid shift, together with a closest approach, during the lifetime of the mission. This is not the actual number of events that can be extracted from the GAIA dataset, as the false detection rate has not been assessed.

A covariance analysis is used to study the propagation of errors and the estimation of parameters from realistic sampling of the GAIA datastream of transits in the alongscan direction during microlensing events. The mass of the lens can be calculated to good accuracy if the lens is nearby so that angular Einstein radius $\theta_{\mathrm{E}}$ is large; if the Einstein radius projected onto the observer plane $\tilde{r}_{\mathrm{E}}$ is about an astronomical unit; if the duration of the astrometric event is long ( $\gtrsim 1$ year) or if the source star is bright. Monte Carlo simulations are used to study the $\sim 2500$ events for which the mass can be recovered with an error of $<50 \%$. These high quality events are dominated by disk lenses within a few tens of parsecs and source stars within a few hundred parsecs. We show that the local mass function can be recovered from the high quality sample to good accuracy. GAIA is the first instrument with the capabilities of measuring the mass locally in very faint objects like black holes and very cool white and brown dwarfs.

For only $\sim 5 \%$ of all astrometric events will GAIA record even one photometric datapoint. There is a need for a dedicated telescope that densely samples the Galactic Centre and spiral arms, as this can improve the accuracy of parameter estimation by a factor of $\sim 10$. The total number of sources that have an astrometric microlensing variation exceeding the mission target accuracy is $\sim 10^{5}$. The positional measurement of one source in every twenty thousand is affected by microlensing noise at any instant. We show that microlensing is negligible as an unbiased random error source for GAIA.
\end{abstract}

Key words: gravitational lensing - astrometry - Galaxy: stellar content - Galaxy: structure - dark matter

\section{INTRODUCTION}

GAIA is the European Space Agency (ESA) satellite now selected as a Cornerstone 6 mission as part of the Science Program 冈. It is a survey satellite that provides multi-colour, multi-epoch photometry, astrometry and spectroscopy on all objects brighter then $V \approx 20$ (e.g., ESA 2000; Perryman et al. 2001). The dataset is huge with information of un-

\footnotetext{
* http://astro.estec.esa.nl/gaia
}

precedented precision on over a billion objects in our Galaxy alone.

GAIA is the successor to the pioneering Hipparcos satellite, which flew from 1989 to 1993. The Hipparcos program was both simpler and smaller: it measured only $10^{5}$ rather than $10^{9}$ objects and it was provided with a target list rather than being left to determine its targets for itself, as GAIA will. The Hipparcos final results were released only when the mission was complete, whereas many of the science goals of GAIA, especially for bursting or time-varying phenomena

(C) 0000 RAS 


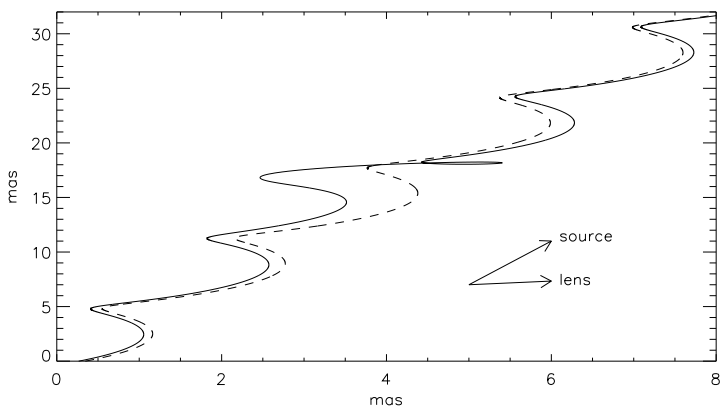

Figure 1. This shows the relative right ascension and declination of a source with (solid line) and without (dashed line) a microlensing event. The trajectory is shown over the GAIA mission lifetime of 5 years. Note that the deviations caused by microlensing are present well before and after the time of maximum of the event. (The lens is at $150 \mathrm{pc}$, the source at $1.5 \mathrm{kpc}$, the transverse velocity is $70 \mathrm{~km} \mathrm{~s}^{-1}$ and the impact parameter $u$ is 1.5 . The lens has mass $0.5 M_{\odot}$. )

like microlensing, may require an early analysis and release of preliminary data.

GAIA carries out continuous scanning of the sky. The satellite rotates slowly on its spin axis, which itself precesses at a fixed angle to the Sun of $55^{\circ}$. As GAIA rotates, light enters the entrance chambers, reflects off mirrors and falls on the focal planes of one of three telescopes, two of which (ASTRO-1, ASTRO-2) measure the positions of stars, one of which (SPECTRO) performs spectroscopy. GAIA observes in three directions along a great circle simultaneously. The astrometric parameters of stars are recovered from the time series of the one-dimensional transits distributed over the five year mission lifetime.

A small fraction of the objects monitored by GAIA will show evidence of microlensing. GAIA can observe microlensing by measuring the photometric amplification of a source star when a lens and a source are aligned. This is the approach followed by the large ground-based microlensing surveys like MACHO, EROS, OGLE and POINT-AGAPE (see e.g., Alcock et al. 1997; Aubourg et al. 1995; Udalski et al. 1994, Aurière et al. 2001). GAIA is inefficient at discovering photometric microlensing events, as the sampling of individual objects is relatively sparse (there are a cluster of observations once every two months on average).

However, there is a much more powerful strategy available to GAIA. Although the two images of a microlensed source are unresolvable, GAIA can measure the small deviation (of the order of a fraction of a milliarcsec) of the centroid of the two images around the trajectory of the source. Astrometric microlensing is the name given to this excursion of the image centroid. The cross section of a lens is proportional to the area it sweeps out on the sky, and so to the product of lens proper motion and angular Einstein radius. Each of these varies as the inverse square root of lens distance, so the signal is dominated by nearby lenses. The detection of astrometric microlensing events for pointed observations has been considered many times before (e.g., Walker 1995; Miralda-Escudé 1996; Paczyński 1996; Boden, Shao \& van Buren 1998). Once alerted by a ground-based survey, the events are followed by narrow angle differential astrometry. The detection of astrometric microlensing using observations from a scanning satellite has been considered before by Høg, Novikov \& Polnarev (1995) in the context of the proposed ROEMER mission (a forerunner of GAIA).

We will show that the all-sky source-averaged astrometric microlensing optical depth is $\sim 2.5 \times 10^{-5}$, over an order of magnitude greater than the photometric microlensing optical depth. There are two main difficulties facing GAIA in exploiting this comparatively high probability. First, the astrometric accuracy of a single measurement by GAIA depends on the source magnitude and quickly degrades at magnitudes fainter than $G \approx 15$. Second, GAIA provides a timeseries of one-dimensional astrometry. The observed quantity is the CCD transit time for the coordinate along the scan. This is the same way the Hipparcos satellite worked (see The Hipparcos and Tycho Catalogues, ESA (1997), volume 3 , section 16). From the sequence of these one-dimensional measurements, the astrometric path of the source, together with any additional deflection caused by microlensing, must be recovered.

This paper assesses the microlensing signal that will be seen by GAIA. Section 2 gives the formulae for the photometric and astrometric microlensing optical depths. These are used to estimate the total number of microlensing events that GAIA can measure. In Section 3, a covariance analysis is used to demonstrate the effects of error propagation on the recovery of the parameters of microlensing events. For the purposes of GAIA, we show that the disk stars within a few hundred parsecs of the Sun are the most important source and lens populations. Section 4 describes the extinction law and the Galaxy model used to generate microlensing events for our Monte Carlo simulations. The synthetic data are sampled with GAIA's scanning law and realistic errors are applied to provide the one-dimensional astrometric datastreams. Section 5 discusses the results of the simulations, both for the entire sample of events and for the subset of gold-plated events whose parameters can be recovered to good accuracy. Finally, Section 6 examines the overall strategy for identification of events, which need to be distinguished from other forms of astrometric deviation such as binary companions.

\section{ASTROMETRIC MICROLENSING}

\subsection{General Principles}

Let us consider the lensing of a luminous source by a dark point lens. (Of course, the lens may be luminous, but we defer consideration of this till Section 6). The angular position of the source $\boldsymbol{\theta}_{\mathrm{s}}$ can be written as

$\boldsymbol{\theta}_{\mathrm{s}}(t)=\boldsymbol{\theta}_{\mathrm{s}, 0}+\boldsymbol{\mu}_{\mathrm{s}} t+\mathbf{P}_{\mathrm{s}}$,

where $\boldsymbol{\theta}_{\mathrm{s}, 0}$ is the zero-point, $\boldsymbol{\mu}_{\mathrm{s}}$ is the source proper motion and $\mathbf{P}_{\mathrm{s}}$ is the source parallax. Similarly, the angular separation between source and lens is given by

$\boldsymbol{\theta}_{\mathrm{sl}}(t)=\boldsymbol{\theta}_{\mathrm{sl}, 0}+\boldsymbol{\mu}_{\mathrm{sl}} t+\mathbf{P}_{\mathrm{sl}}$,

where the proper motion $\boldsymbol{\mu}_{\mathrm{sl}}$ and parallax $\mathbf{P}_{\mathrm{sl}}$ are of the source relative to the lens.

Microlensing induces an additional shift of the source centroid. Letting $\mathbf{S}(t)$ be the image centroid, we have (e.g., Walker 1995; Dominik \& Sahu 2000) 


\begin{tabular}{cccccccccccc}
\hline$G$ (in mag) & 10 & 11 & 12 & 13 & 14 & 15 & 16 & 17 & 18 & 19 & 20 \\
$\sigma_{\mathrm{p}}$ (in mmag) & 9 & 9 & 9 & 9 & 9 & 10 & 12 & 16 & 23 & 36 & 60 \\
$\sigma_{\mathrm{a}}$ (in $\mu$ as) & 30 & 30 & 30 & 40 & 60 & 90 & 150 & 230 & 390 & 700 & 1400 \\
\hline
\end{tabular}

Table 1. This table lists the mean accuracy in photometry $\sigma_{\mathrm{p}}$ and in position $\sigma_{\mathrm{a}}$ versus $G$ band magnitude for the GAIA satellite. Note that $\sigma_{\mathrm{a}}$ is the accuracy of a single astrometric measurement, not the target accuracy at the end of the GAIA mission, which is better by a factor of $\sim 10$. The values are approximate sky averages, adapted from Tables 7.3 and 8.2 of ESA (2000).
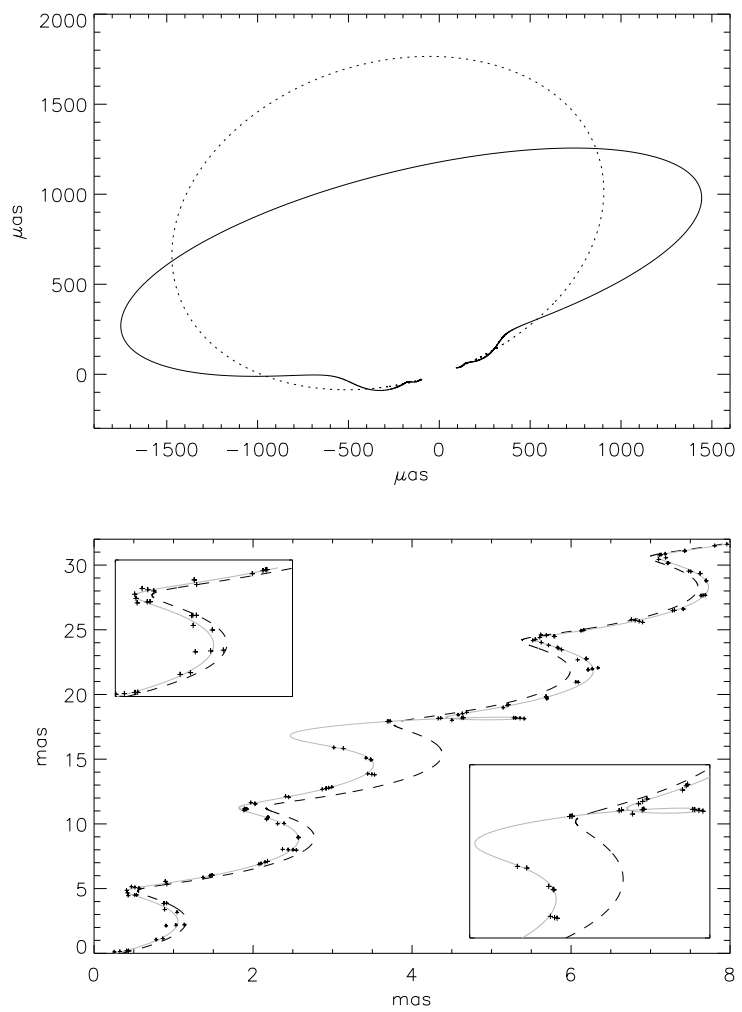

Figure 2. Upper panel: Astrometric shift of the microlensing event of Figure 1, as seen by a barycentric (dotted line) and a terrestrial observer (solid line). Lower panel: Simulated data incorporating typical sampling and astrometric errors for GAIA. Also shown for comparison are the theoretical trajectories of the source with (grey line) and without (dashed line) the event. The insets show the deviations at the beginning and the midpoint of this high signal-to-noise event. (The accuracy $\sigma_{\mathrm{a}}$ of the astrometry is $300 \mu$ as, corresponding roughly to a 17 th magnitude star).

$$
\mathbf{S}(t)=\boldsymbol{\theta}_{\mathrm{s}}(t)+\boldsymbol{\theta}(t), \quad \boldsymbol{\theta}=\frac{\boldsymbol{\theta}_{\mathrm{sl}}(t)}{\left(\theta_{\mathrm{sl}}(t) / \theta_{\mathrm{E}}\right)^{2}+2} .
$$

Here, the angular Einstein radius $\theta_{\mathrm{E}}$ is related to the lens mass $M$ by

$$
\frac{\theta_{\mathrm{E}}}{\operatorname{mas}}=\left(\frac{M}{0.12 M_{\odot}}\right)^{1 / 2}\left(\frac{\pi_{\mathrm{sl}}}{\operatorname{mas}}\right)^{1 / 2}
$$

where $\pi_{\mathrm{sl}}=\left|\mathbf{P}_{\mathrm{sl}}\right|$. Figure 1 shows the astrometric trajectory of the same source with and without a microlensing event. In this case, the lens is just $150 \mathrm{pc}$ away from the observer, while the source is a disk star $1.5 \mathrm{kpc}$ away. The microlensing event causes the additional excursion superimposed on the parallactic and proper motion of the source.
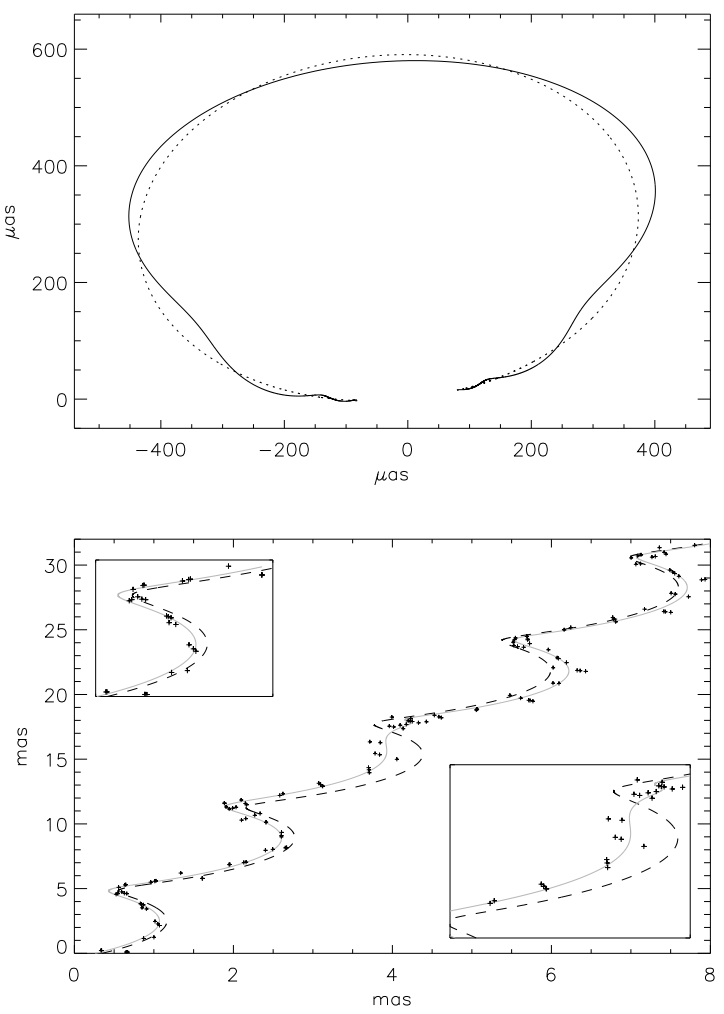

Figure 3. Same as Figure 2, but the lens distance is $1 \mathrm{kpc}$. This shows a microlensing event with a $5 \sqrt{2} \sigma_{\text {a }}$ variation of the centroid shift. GAIA will be able to measure the astrometric deviation but will not be able to extract any useful information on the microlensing parameters.

The characteristic lengthscale in microlensing is the Einstein radius $R_{\mathrm{E}}=D_{\mathrm{l}} \theta_{\mathrm{E}}$. It is the linear size of the angular Einstein radius in the lens plane. For applications in the Galaxy, we find that

$R_{\mathrm{E}}=9 \mathrm{au} \sqrt{\frac{M}{M_{\odot}}} \sqrt{\frac{D_{\mathrm{l}}}{10 \mathrm{kpc}}} \sqrt{1-\frac{D_{\mathrm{l}}}{D_{\mathrm{s}}}}$,

and so $R_{\mathrm{E}}$ is of the typical size of a few astronomical units (aus). Here, $D_{1}$ and $D_{\mathrm{s}}$ are the distances from the observer to the lens and source respectively. The characteristic timescale in photometric microlensing is the Einstein crossing time $t_{\mathrm{E}}$, which is the time taken for the source to cross the Einstein radius. 

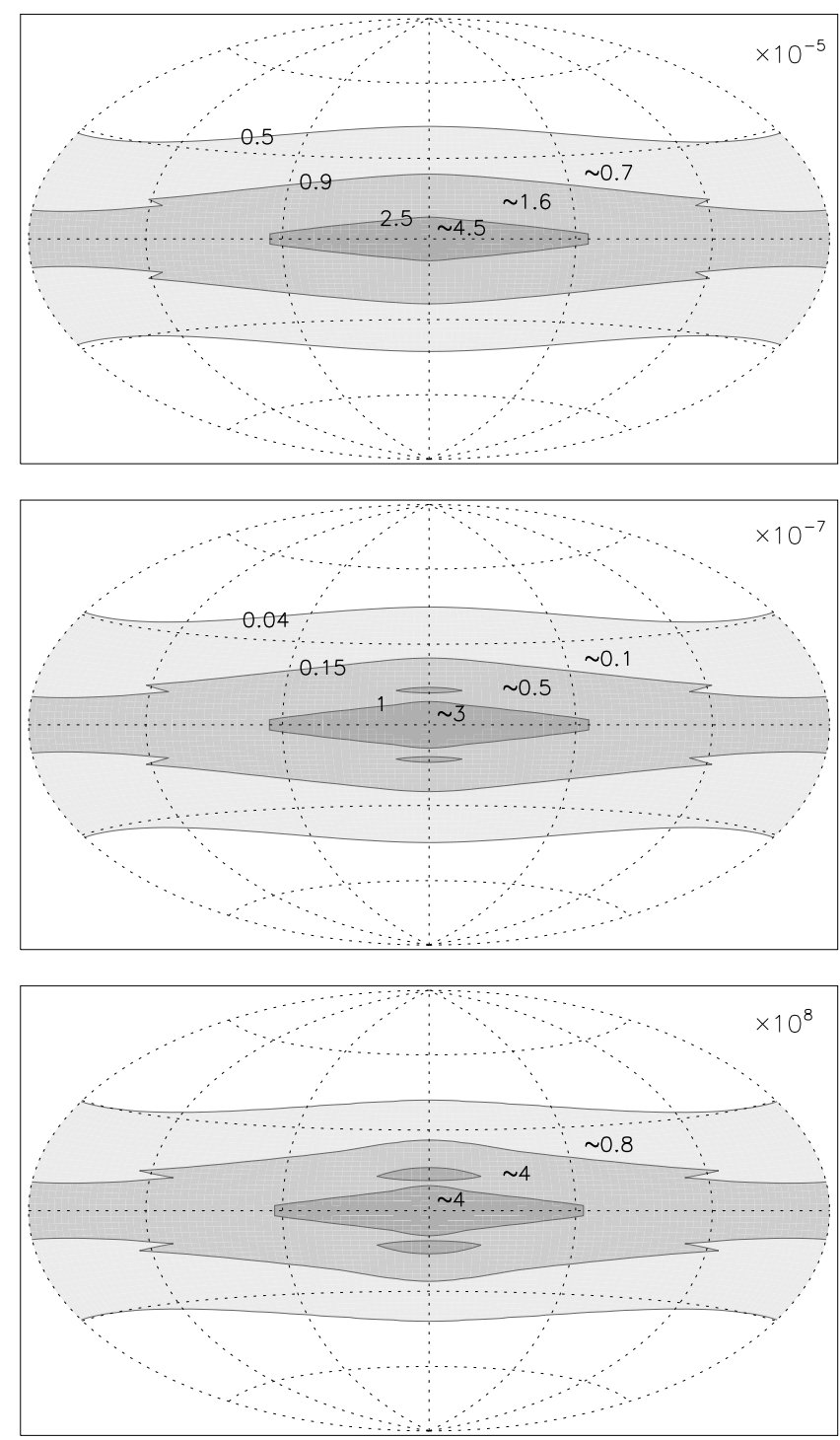

Figure 4. Upper panel: An all-sky map of the source-averaged astrometric optical depth. Meridians of galactic latitude are shown at $60^{\circ}$ intervals, parallels of longitude at $30^{\circ}$ intervals. The contour levels are marked. The number within each contour refers to the mean value of the optical depth inside the shaded region enclosed between the contours. All numbers are in units of $10^{-5}$. Middle panel: As the upper panel, but for the photometric microlensing optical depth. All numbers are in units of $10^{-7}$. Lower panel: An all-sky map of the starcounts. The number within each contour refers to the total number of stars inside the shaded region enclosed between the contours. To find the instantaneous number of events in any region, we multiply the number of stars by the average optical depth. (For details of the source and lens populations and extinction model, see Section 4).

\subsection{The Centroid Shift}

The centroid shift points away from the dark lens as seen by the source. The dimensionless function

$\mathbf{u}=\frac{\boldsymbol{\theta}_{\mathrm{sl}}}{\theta_{\mathrm{E}}}$

is the angular separation in units of the angular Einstein radius. For $u \rightarrow \infty$, the centroid shift falls off like
$S=|\mathbf{S}| \sim \frac{\theta_{\mathrm{E}}}{u}$.

This can be compared with the photometric magnification $A$, which falls off like

$A \sim 1+\frac{2}{u^{4}}$.

These asymptotic results illustrate one of the important differences between astrometric and photometric microlensing. The centroid shift falls off much more slowly than the magnification, so that the cross-section for astrometric events is much larger than for photometric events (e.g., Paczyński 1996; Miralda-Escudé 1996).

In the absence of the proper and parallactic motion, the absolute value of the centroid shift is

$\theta=\frac{\sqrt{u_{0}^{2}+\hat{t}^{2}}}{u_{0}^{2}+\hat{t}^{2}+2} \theta_{\mathrm{E}}$.

Here, $u_{0}$ is the value of the dimensionless distance at the time of closest approach $t_{0}$ and

$\hat{t}(t)=\frac{t-t_{0}}{t_{\mathrm{E}}}=\frac{\mu_{\mathrm{sl}}\left(t-t_{0}\right)}{\theta_{\mathrm{E}}}$.

The shift can be decomposed into components parallel and perpendicular to the direction of motion of the lens relative to the source, namely

$\theta_{\|}=\frac{\hat{t}}{u_{0}^{2}+\hat{t}^{2}+2} \theta_{\mathrm{E}}, \quad \theta_{\perp}=\frac{u_{0}}{u_{0}^{2}+\hat{t}^{2}+2} \theta_{\mathrm{E}}$.

As pointed out by Walker (1995), the centroid shift as seen by a barycentric observer is an ellipse. For $u \rightarrow 0$, the ellipse becomes a straight line, while for $u \rightarrow \infty$, it becomes a circle.

Figure 2 shows two further views of the same microlensing event presented in Figure 1. The upper panel shows the right ascension and declination recorded by a barycentric and a terrestrial observer (or equivalently a satellite at the $\mathrm{L}_{2}$ Lagrange point, like GAIA). The proper and parallactic motion of the source have been subtracted out. However, GAIA does not provide the data in such a clean form, as it really measures a series of one-dimensional transits of the two-dimensional astrometric curve. The lower panel shows the event as seen by GAIA. The simulated datapoints have been produced by generating random transit angles, and sampling the astrometric curve according to GAIA's scanning law for the ASTRO-1 and ASTRO-2 telescopes (using programs freely available from L. Lindegren at Lund Observatory). The transits are strongly clustered, as GAIA spins on its axis once every 3 hours and so may scan the same patch of sky four or five times a day. The transit angle is the same for all transits in such a cluster, but changes randomly from cluster to cluster. Gaussian astrometry errors with standard deviation $\sigma_{\mathrm{a}}=300 \mu$ as have been added to the simulated datapoints. The two insets show the astrometric deviations at the beginning and at the maximum of the event, from which it is clear that GAIA can detect that a microlensing event has occurred. Figure 3 shows the same microlensing event, except that the lens distance has been increased. This causes the relative parallax $\pi_{\mathrm{sl}}$, and consequently the angular Einstein radius $\theta_{\mathrm{E}}$, to diminish. The upper panel of Figure 3 shows that the parallactic deviation of the curve is also smaller than before. It is already clear from the lower panel that it will be difficult to extract phys- 


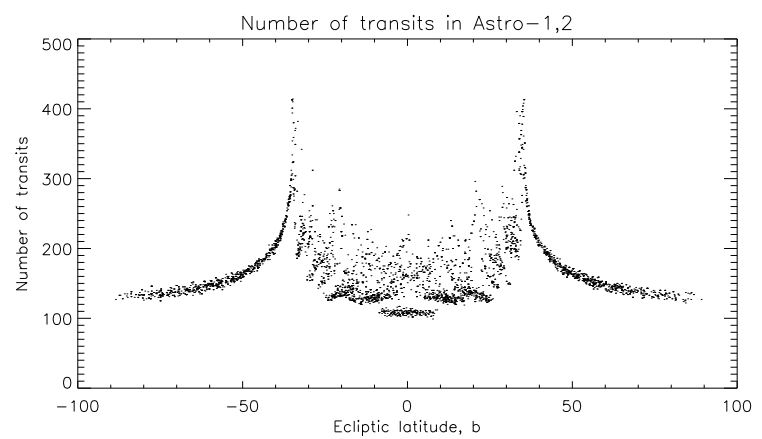

Figure 5. This shows the number of transits made by the ASTRO-1 and ASTRO-2 telescopes as a function of ecliptic latitude. The figure is drawn by choosing 5000 random directions on the sky, and computing the ecliptic latitude and number of transits using software supplied by L. Lindegren. It should be remembered that the number of transits may be misleading, as the transits are strongly clustered into groups of between two and five.

ical information from some of the events, particularly those with smaller Einstein radius.

\subsection{Optical Depths}

The probability $\tau$ of detecting a microlensing event with certain characteristics is just

$\tau=D_{\mathrm{s}} \int_{0}^{1} d x \int_{0}^{\infty} d M \frac{\rho(x)}{M} \Sigma(x, M) f(M)$,

where $\Sigma$ is the area in the lens plane for which the projected source positions yield the required effect and $f(M)$ is the mass function or the number of lenses with mass in the range $M$ to $M+d M$.

GAIA measures displacements only along the scan, so we incorporate a factor of $\sqrt{2}$ to take us from GAIA's onedimensional astrometric accuracies to the two-dimensional accuracies. The centroid shift varies by more than $5 \sqrt{2} \sigma_{\mathrm{a}}$ when the projected position of the source lies within a circle of radius $u_{\mathrm{a}} R_{\mathrm{E}}$, where (Dominik \& Sahu 2000)

$u_{\mathrm{a}}=\sqrt{\frac{T_{\text {life }} v}{5 \sqrt{2} \sigma_{\mathrm{a}} D_{\mathrm{l}}}}=\sqrt{\frac{T_{\text {life }} \theta_{\mathrm{E}}}{5 \sqrt{2} \sigma_{\mathrm{a}} t_{\mathrm{E}}}}$.

Here, $T_{\text {life }}$ is the lifetime of the satellite and $v$ is the transverse source velocity in the lens plane. Note that $5 \sqrt{2} \sigma_{\mathrm{a}}$ is enough for GAIA to make a convincing measurement of the shift, but not usually enough for detection of a microlensing event.

Instead, the minimum criterion needed to detect the event in the GAIA database is a variation of centroid shift larger than $5 \sqrt{2} \sigma_{\text {a }}$ together with a closest approach between lens and source happening with a time $T_{\text {life }}$. The area in the lens plane giving rise to events with these characteristics is $\Sigma=2 u_{\mathrm{a}} R_{\mathrm{E}} T_{\text {life }} v$. Using (12), the probability is (Dominik \& Sahu 2000)

$\tau_{\mathrm{a}}=4 \sqrt{\frac{G}{c^{2}}} D_{\mathrm{s}}\left\langle M^{-1 / 2}\right\rangle \sqrt{\frac{T_{\mathrm{life}}^{3} v^{3}}{5 \sqrt{2} \sigma_{\mathrm{a}}}} \int_{0}^{1} d x \rho(x) \sqrt{1-x}$,

where

$$
\left\langle M^{-1 / 2}\right\rangle=\int_{0}^{\infty} M^{-1 / 2} f(M) d M .
$$

We refer to the quantity (14) as the astrometric microlensing optical depth.

By contrast, the probability of a microlensing event with an amplification greater than that corresponding to the threshold of photometric accuracy $5 \sigma_{\mathrm{p}}$ is (e.g., Griest 1991, Dominik \& Sahu 2000), namely

$\tau_{\mathrm{p}}=\frac{4 \pi G}{c^{2}} u_{\mathrm{p}}^{2} D_{\mathrm{s}}^{2} \int_{0}^{1} d x \rho(x) x(1-x)$,

where

$u_{\mathrm{p}}^{2}=\frac{2}{\sqrt{1-A_{\mathrm{p}}^{-2}}}-2$.

Here, $A_{\mathrm{p}}$ is the magnification corresponding to the threshold photometric accuracy $5 \sigma_{\mathrm{p}}$. We refer to the quantity $(16)$ as the photometric microlensing optical depth. For GAIA, both the astrometric and photometric accuracies depend on the magnitude of the source as listed in Table 1.

The Einstein crossing time $t_{\mathrm{E}}$ is the time taken for the source to cross the Einstein radius. This is not the duration of a photometric event, which is the time taken for the source to cross a circle of radius $u_{\mathrm{p}} R_{\mathrm{E}}$ in the lens plane. The duration of an astrometric event is the time for which the centroid shift is greater than the threshold $5 \sqrt{2} \sigma_{\mathrm{a}}$. Dominik \& Sahu (2000) show that this is approximately the time taken to cross a circle of radius $R_{\mathrm{E}} \theta_{\mathrm{E}} /\left(5 \sqrt{2} \sigma_{\mathrm{a}}\right)$. So, the duration of an astrometric event is

$t_{\mathrm{ae}}=t_{\mathrm{E}} \frac{\theta_{\mathrm{E}}}{5 \sqrt{2} \sigma_{\mathrm{a}}}$.

For GAIA, this is typically a factor of two times longer than the duration of a photometric event.

Figure 1 show contours of astrometric and photometric microlensing optical depth together with starcounts. The maps assume a standard model for the sources and lenses in the Galaxy, together with a luminosity function and extinction law (described in Section 4). They have been produced assuming that the relative source-lens velocity in the lens plane is $\sim 140 \mathrm{~km} \mathrm{~s}^{-1}$ (see Figure 12). Extinction is an important effect for GAIA's microlensing capabilities, as the accuracy of both the astrometry and photometry depends on source magnitude. Taking into account the effects of extinction, the all-sky averaged value of the astrometric optical depth is $2.5 \times 10^{-5}$. Here, the averaging is performed by weighting the optical depth with the starcount density. Regions like the Galactic bulge are very heavily weighted, and so the average astrometric optical depth is of the same order of magnitude as the typical optical depth towards the central part of the Galaxy. There are $\sim 10^{9}$ stars brighter than $V=20$ in our model; the same is true for the Galaxy (Mihalas \& Binney 1981). This means that, during the GAIA mission, there are $\sim 25000$ astrometric microlensing events, which have a variation of the centroid shift greater than $5 \sqrt{2} \sigma_{\text {a }}$ together with a closest approach during the mission lifetime. This number can be read off Figure 7 , by multiplying the mean astrometric optical depth within the central contour of the upper panel by the total number of stars within the central contour of the lower panel. Some of these displacements cannot be identified by GAIA as microlensing events, first because the signal-to-noise will often be low 
and second because any identification algorithm must not generate too many false detections. The middle panel of Figure 4 show contours of photometric microlensing optical depth again including the effects of extinction. The all-sky averaged photometric optical depth is $\sim 1.2 \times 10^{-7}$. Let us recall that, from eqs (16 17 ), a photometric event occurs whenever there is $5 \sigma_{\mathrm{p}}$ variation. The typical event duration is $\sim 2$ months, so that there are a total of $\sim 3600$ photometric microlensing events during the GAIA mission. However, a very substantial number of these events will be undetectable. GAIA's sampling is sparse compared to ground-based programs like MACHO, EROS or POINT-AGAPE, so many of these events will be missed.

\section{ERROR PROPAGATION}

\subsection{Covariance Analysis}

Our first task is to understand the propagation of errors, so that we can assess how many of the $\sim 25000$ potential astrometric microlensing events are useful.

Since most events will not be detected photometrically by GAIA, it will not usually be possible to combine photometric together with astrometric data to analyze a microlensing event. The measured quantity that will generally be provided by GAIA is the source displacement along the scan. This contains information on the microlensing event, but is contaminated with the source parallactic and proper motion as well. (We assume that the GAIA datastream has already been corrected for aberration due to satellite motion and gravitational deflection caused by Solar System objects).

The components of $\mathbf{S}$ resolved with respect to right ascension $\alpha$ and declination $\delta$ are

$$
\left(S_{\alpha}, S_{\delta}\right)=\boldsymbol{\theta}_{\mathrm{s}}+\frac{\mathbf{u}}{u^{2}+2} \theta_{\mathrm{E}} .
$$

The angular position of the source $\boldsymbol{\theta}_{\mathrm{s}}$ contains the contributions from the source proper motion $\boldsymbol{\mu}_{\mathrm{s}}$ and source parallax $\mathbf{P}_{\mathrm{s}}$. We note that

$\mathbf{u}=\left(\begin{array}{c}\hat{t} \cos \phi-u_{0} \sin \phi-\pi_{\mathrm{sl}} P_{\alpha} \theta_{\mathrm{E}}^{-1} \\ \hat{t} \sin \phi+u_{0} \cos \phi-\pi_{\mathrm{s} 1} P_{\delta} \theta_{\mathrm{E}}^{-1}\end{array}\right)$

where $\phi$ is the proper motion angle (Gould \& Salim 1999). We have also expanded the parallaxes $\mathbf{P}_{\mathrm{s}}$ and $\mathbf{P}_{\mathrm{sl}}$ as

$\mathbf{P}_{\mathrm{s}}=\pi_{\mathrm{s}}\left(P_{\alpha}, P_{\delta}\right), \quad \mathbf{P}_{\mathrm{sl}}=\pi_{\mathrm{sl}}\left(P_{\alpha}, P_{\delta}\right)$,

so that $\pi_{\mathrm{s}}$ and $\pi_{\mathrm{sl}}$ are the absolute values and $P_{\alpha}$ and $P_{\delta}$ are the direction cosines (given in van der Kamp 1967).

The directions along and perpendicular to the scan are related to right ascension and declination by a rotation, which depends on where the satellite is pointing. As GAIA continuously scans the sky, this angle can take any value for the same source. So, formulae (19)-(21) hold good in the scan coordinate system as well, after rotation by a random angle to take us from the right ascension and declination to the along-scan and across-scan coordinates. GAIA measures positions in the along-scan direction only.

We use this microlensing model to create synthetic astrometric measurement sets. There are a total of 11 parameters which we wish to compute from the data, namely: $\boldsymbol{\mu}_{\mathrm{s}}$,

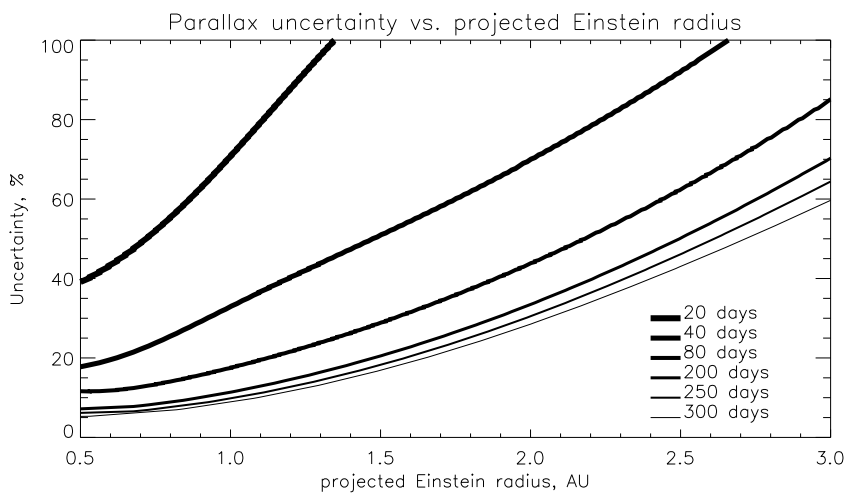

Figure 6. This shows the percentage error in estimation of the relative parallax as a function of the projected Einstein radius $\tilde{r}_{\mathrm{E}}$ for different astrometric event durations and a given accuracy $\sigma_{\mathrm{a}}=150 \mu \mathrm{as}$. The events have varying source and lens distance, as well as the transverse velocity. (All the other parameters are as in Figure 1, except $u=1$ ).

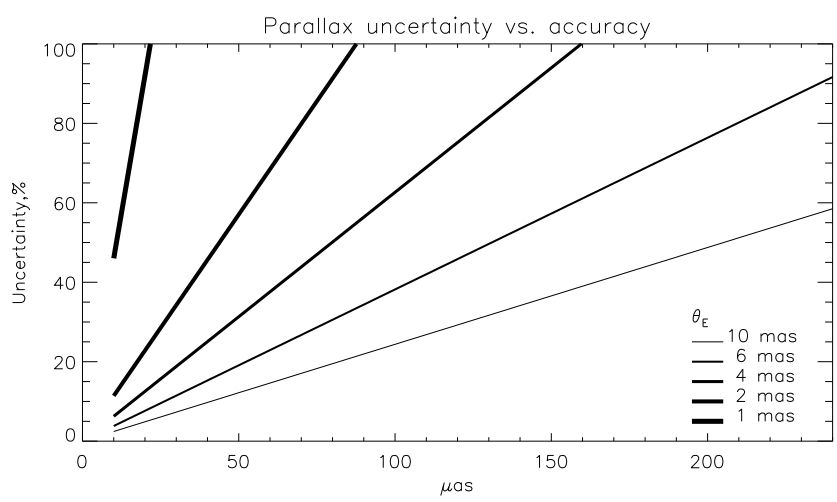

Figure 7. This shows the percentage error in estimation of the relative parallax as a function of astrometric accuracy for different angular Einstein radii $\theta_{\mathrm{E}}$. We vary the Einstein radius by varying the source and lens distance at fixed transverse velocity. (All the other parameters are as in Figure 1, except $u=5$ ).

$\pi_{\mathrm{s}}, u_{0}, t_{0}, t_{\mathrm{E}}, \phi, \theta_{\mathrm{E}}, \pi_{\mathrm{sl}}$ and $\boldsymbol{\theta}_{\mathrm{s}, 0}$. To study parameter estimation performance, we evaluate the covariance matrix $c_{i j}$ (see e.g., Boutreux \& Gould 1996; Gould \& Salim 1999). This is defined as the matrix $\mathbf{C}=\mathbf{B}^{-1}$, where

$b_{i j}=\sum_{k=1}^{N} \sigma_{\mathrm{a}}^{-2} \frac{\partial \theta_{\alpha}\left(t_{k}\right)}{\partial a_{i}} \frac{\partial \theta_{\alpha}\left(t_{k}\right)}{\partial a_{j}}$.

Diagonal elements of the matrix $\sqrt{c_{i i}}$ are the individual uncertainties in the parameters $a_{i}$ assuming the standard $\chi^{2}$ fitting procedure. Here, $a_{1}, \ldots, a_{11}$ are the 11 parameters stated above and $\sigma_{\mathrm{a}}$ is the astrometric accuracy for a given star, which depends on the source magnitude and is recorded in Table 1 .

Note that $t_{k}$ are the times at which GAIA samples the one-dimensional astrometry datastream. In our calculations, these times are always generated from GAIA's scanning law using Lindegren's software. GAIA's scanning law is quite complicated, as shown in Figure 5. The number of times GAIA samples an object $N$ is between 100 and 450, depending on its ecliptic latitude (Lindegren 1998). The number of 


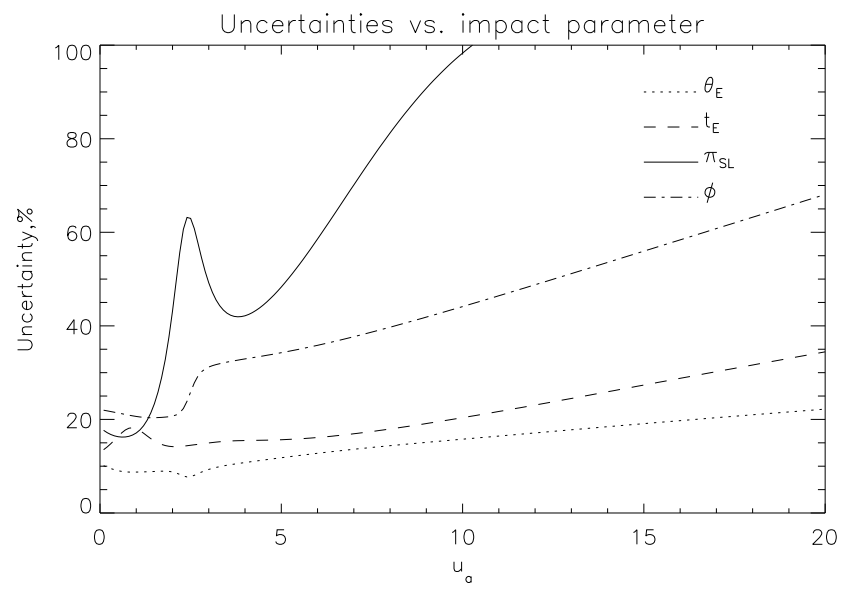

Figure 8. This shows the percentage error in estimation of the microlensing parameters as a function of impact parameter $u$. Accurate recovery of the relative parallax $\pi_{\mathrm{sl}}$ and the proper motion angle $\phi$ is harder, whereas recovery of the angular Einstein radius $\theta_{\mathrm{E}}$ and the Einstein crossing time $t_{\mathrm{E}}$ is easier. (The event has the same parameters as Figure 1 with $\sigma_{\mathrm{a}}=150 \mu$ as.)

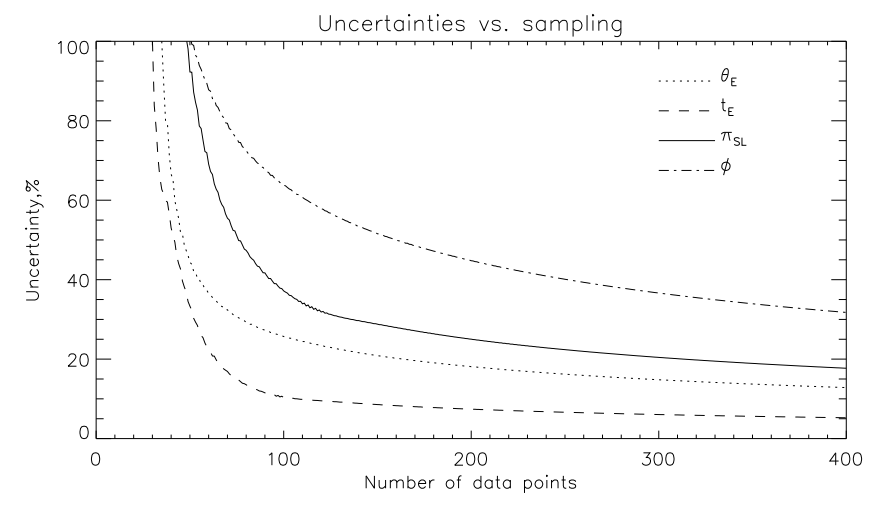

Figure 9. This shows the percentage error as a function of number of datapoints for a typical microlensing event. Provided there are $\gtrsim 150$ measurements, then the sampling is not the critical factor. (The event has the same parameters as Figure 1, but $\left.\sigma_{\mathrm{a}}=100 \mu \mathrm{as}\right)$.

transits is greatest, and so the final astrometry is most accurate, for objects close to an ecliptic latitude $\sim 35^{\circ}$. For a typical location, the number of transits is between 100 and 200. These transits are usually clustered into groups of between 2 and 5, so the average effective number of independent datapoints is $\sim 40$.

\subsection{Errors in Estimates of the Astrometric Microlensing Parameters}

Although the relative parallax $\pi_{\mathrm{sl}}$ is quite hard to estimate, it is valuable since it provides a unique solution for the mass of the lens $M$ when combined with $\theta_{\mathrm{E}}$ (see eq. (4)). If in addition the source distance $D_{\mathrm{s}}$ can be estimated - for example, if GAIA itself measures the source parallax - then we have a complete solution for all the microlensing parameters. Let us recall that in the ground-based MACHO, EROS and OGLE programs, there is not a single microlensing event for which the parameters can be derived unambiguously from the data alone. Consequently, the location of the lenses responsible for the events towards the Magellanic Clouds is a matter of considerable controversy (e.g., Sahu 1994, Gould 1995, Alcock et al. 1997, Evans \& Kerins 2000, Zhao \& Evans 2000). GAIA offers the promise of providing a sample of lensing events for which all the parameters can be extracted without any modelling assumptions. However, some caution is needed, as we already known in the context of the Space Interferometry Mission (SIM) by Gould \& Salim (1999) that astrometric measurements alone do not necessarily guarantee accurate estimation of the relative parallax.

For which events can we recover the relative parallax to good accuracy from the GAIA data? First of all, an event must last an adequate time for the microlensing shift to be distorted by parallactic movement of the lens. Thus, the error will depend on the duration of the astrometric event. Second, the amplitude of the distortion is dictated by the Einstein radius projected onto the observer plane (e.g., Gould \& Salim 1999, Gould 2000), namely

$\tilde{r}_{\mathrm{E}}=\frac{\theta_{\mathrm{E}}}{\pi_{\mathrm{sl}}}$.

For close lenses $\left(D_{1} \approx 100 \mathrm{pc}\right)$, it follows that $1-D_{\mathrm{l}} / D_{\mathrm{s}} \rightarrow 1$, and so the projected Einstein radius becomes

$\tilde{r}_{\mathrm{E}} \approx R_{\mathrm{E}} \approx 1 \mathrm{au} \sqrt{\frac{M}{M_{\odot}}} \sqrt{\frac{D_{\mathrm{l}}}{100 \mathrm{pc}}}$.

For a measurable distortion, we require $\tilde{r}_{\mathrm{E}}$ to be about an astronomical unit or smaller. If it is too large, then the Earth's motion about the Sun has a negligible effect. Accuracy in the relative parallax is a trade-off between duration and lens distance.

Figure 6 shows the uncertainty in $\pi_{\mathrm{sl}}$ as a function of $\tilde{r}_{\mathrm{E}}$ for different event durations $t_{\mathrm{ae}}$, while the companion Figure 7 shows the uncertainty in $\pi_{\mathrm{sl}}$ as a function of astrometric accuracy for different $\theta_{\mathrm{E}}$. It aids accurate recovery of the relative parallax if the angular Einstein radius is large. The error in $\pi_{\mathrm{sl}}$ degrades for distant lenses. The degradation is worse for the shorter duration events. Note that the close lenses $\left(D_{1} \approx 100 \mathrm{pc}\right)$ typically have a crossing time $t_{\mathrm{E}} \approx 1 \mathrm{au} / 150 \mathrm{kms}^{-1} \approx 10$ days, and so the astrometric event duration is typically 200 days. It is the close lenses with longer timescales that provide the most propitious circumstances for measuring $\pi_{\mathrm{sl}}$ from the data.

Figure 8 shows the uncertainties in $\theta_{\mathrm{E}}, t_{\mathrm{E}}, \pi_{\mathrm{sl}}$ and $\phi$ as a function of the dimensionless impact parameter. A typical value of $u$ for GAIA is 11 or so. For large $u$, the parallactic distortion is superposed upon a small eccentricity ellipse (see eq. (11)). For small $u$, the eccentricity of the ellipse becomes large and the parallactic distortion makes the curve thinner and more elongated. This can be an almost degenerate situation for estimating the microlensing parameters from one-dimensional astrometry. The percentage error in $\phi$ and $\pi_{\mathrm{sl}}$ is larger than the error in $\theta_{\mathrm{E}}$ and $t_{\mathrm{E}}$. This is because $\pi_{\mathrm{sl}}$ controls the shape of the astrometric curve, $\phi$ controls the orientation, while $\theta_{\mathrm{E}}$ and $t_{\mathrm{E}}$ the size. Given one-dimensional astrometry, it is more difficult to recover the details of the shape than the size. The rise and fall in error at $u \approx 3$ in Figure 8 is a consequence of the degeneracy of the one-dimensional astrometry. Asymptotically, the percentage error always increases with increasing 
impact parameter. However, there is a régime at small impact parameter when the converse is true. The reason for this is as follows. For very close lenses, the parallactic distortion can become so large that the direction of traversal of the astrometric curve is reversed. This degeneracy is inherent in microlensing and it is not a consequence of the limited information that can be extracted from measurements. The astrometric curve can now correspond to events with positive impact parameter and large parallactic effect, or negative impact parameter and small parallactic effect. There is no way to tell the difference.

Finally, Figure 9 shows the effects of sampling. The error in selected microlensing parameters is plotted against the number of datapoints for a typical microlensing event. In this Figure only, the sampling is not performed according to GAIA's sampling law, but the datapoints are chosen uniformly over the mission lifetime, as we wish to illustrate the importance of sampling. In fact, GAIA samples every object between 100 and 450 times depending on ecliptic latitude. So, at first glance, Figure 9 seems to offer reassurance that sampling is not a critical factor, as it shows that the error increases swiftly only once the effective number of datapoints falls below 40. However, this is misleading because GAIA's datapoints are clustered into groups of between 2 and 5 , or typically 4 . This is disadvantageous, as it reduces the effective number of datapoints by $\sim 4$, although the accuracy of the astrometry is improved by $\sim 2$. Figure 9 tells us that if the number of independent datapoints falls below $\sim 40$, then sampling is the limiting factor in the extraction of useful parameters. (Our calculations show that the form of the figure is only weakly dependent on $\sigma_{\text {a }}$ ). In other words, as $\sim 40$ is roughly the average number of effective datapoints, the sampling is responsible for roughly tripling or quadrupling the errors in the estimated parameters for about half the dataset.

\subsection{Errors in Estimates of Masses of Lenses}

We are primarily interested in finding the masses of the lenses. The error in the mass is related to the errors in the angular Einstein radius and the relative parallax by

$$
\left(\frac{\sigma_{M}}{M}\right)^{2}=4 \frac{\sigma_{\theta_{\mathrm{E}}}^{2}}{\theta_{\mathrm{E}}^{2}}+\frac{\sigma_{\pi_{\mathrm{sl}}}^{2}}{\pi_{\mathrm{sl}}}-4 \hat{C}\left(\theta_{\mathrm{E}}, \pi_{\mathrm{sl}}\right) \frac{\sigma_{\theta_{\mathrm{E}}}}{\theta_{\mathrm{E}}} \frac{\sigma_{\pi_{\mathrm{sl}}}}{\pi_{\mathrm{sl}}},
$$

where $\hat{C}$ is the correlation coefficient between $\theta_{\mathrm{E}}$ and $\pi_{\mathrm{sl}}$. Let us recall that the elements of the correlation matrix are customarily defined as

$\hat{C}(i, j)=\frac{C_{i j}}{\sqrt{C_{i i} C_{j j}}}$.

The cross-term is important because errors in $\theta_{\mathrm{E}}$ and $\pi_{\mathrm{sl}}$ are strongly correlated for most of the events. This is a small term for low impact parameter events, but it becomes important once $u_{\mathrm{a}}>2$. The cross-term always decreases the uncertainty in the mass. This is why Boden et al. (1998) found that eq. (14) of their paper (which neglects the correlation) tends to overestimate the mass error. We always use the full formula (25) to compute the uncertainty in the mass in our simulations.

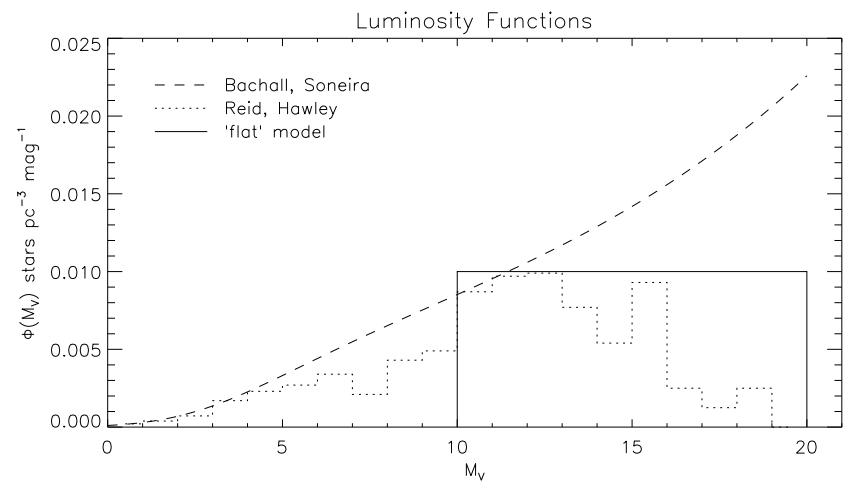

Figure 10. This shows three luminosity functions, namely the Bahcall-Soneira (dashed line), the Reid-Hawley (dotted line) and the flat model (full line). The flat model is the standard one in our simulations.

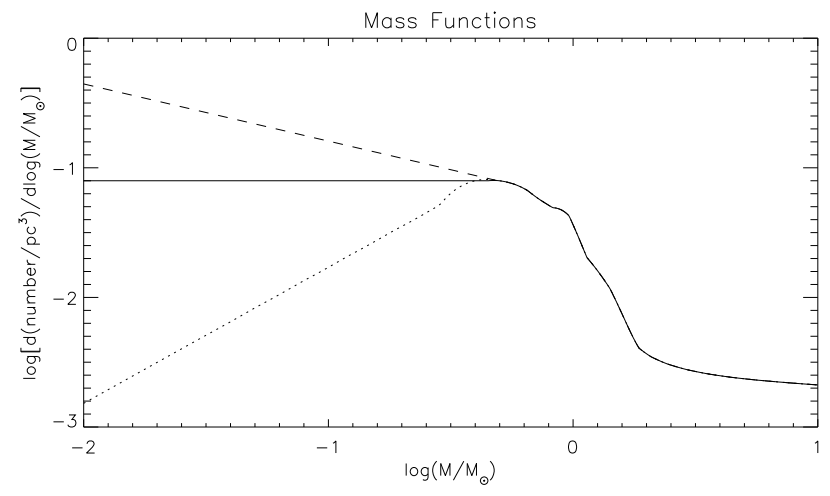

Figure 11. This shows three mass functions that are flat, rising and falling below $0.5 M_{\odot}$. Above $0.5 M_{\odot}$, the mass function is derived from the Reid-Hawley LF. Below $0.5 M_{\odot}$, the mass function cannot be derived with certainty from the observations.

\section{A MODEL OF THE GALAXY}

The goal of the analysis of the microlensing events seen by GAIA is to infer properties of the lenses. The likely results can best be judged by Monte Carlo simulations. Before this, we need to develop a model of the lens and source populations in the Galaxy. This section describes the ingredients of the model in turn.

\subsection{Luminosity Function of Sources}

Let the mass density of sources be $\rho$ and the luminosity function (LF) be $\phi$. In an element of solid angle $d \Omega$, the number of stars in a direction $(\ell, b)$ with distances between $D_{\mathrm{s}}$ and $D_{\mathrm{s}}+d D_{\mathrm{s}}$ and with magnitudes between $m_{\mathrm{G}}$ and $m_{\mathrm{G}}+d m_{\mathrm{G}}$ is

$d N=\frac{1}{\rho_{0}} \phi\left(M_{G}\right) d m_{\mathrm{G}} \rho\left(\ell, b, D_{\mathrm{s}}\right) D_{\mathrm{s}}^{2} d D_{\mathrm{s}} d \Omega$.

Here, $\ell$ and $b$ are Galactic coordinates, so that the solid angle $d \Omega$ is just $\cos b d \ell d b$ and $\rho_{0}$ is the local mass density. As usual, absolute magnitude $M_{G}$ is related to apparent magnitude $m_{\mathrm{G}}$ and extinction via

$M_{G}=m_{\mathrm{G}}-5 \log D_{\mathrm{s}}-A\left(D_{\mathrm{s}}, \ell, b\right)+5$, 


\begin{tabular}{ccl}
\hline $\begin{array}{c}\text { Number of stars } \\
\text { (extinction) }\end{array}$ & $\begin{array}{c}\text { Number of stars } \\
\text { (no extinction) }\end{array}$ & Luminosity Function \\
\hline $0.9 \times 10^{9}$ & $5.0 \times 10^{9}$ & flat model \\
$0.9 \times 10^{9}$ & $5.0 \times 10^{9}$ & Reid, Hawley $(2000)$ \\
$1.1 \times 10^{9}$ & $6.1 \times 10^{9}$ & Bahcall, Soneira (1980) \\
\hline
\end{tabular}

Table 2. The total number of stars with $10<G<20$ for each luminosity function, including and excluding the effects of extinction.

where $A\left(D_{\mathrm{s}}, \ell, b\right)$ is the extinction law. Equation (27) can be interpreted as a probability density for the source parameters $D_{\mathrm{s}}, \ell, b$ and $M_{G}$.

Three source LFs are shown in Figure 10. These are valid for the $V$ band, whereas GAIA's photometry is most accurate in the broad $G$ band. As our aim is to provide approximate numbers in our calculations, the conversion from $V$ to $G$ band in the LF will not have a noticeable effect on the results.

The Bahcall-Soneira (1980) LF is a reasonably accurate predictor of the numbers of stars in the Galaxy fainter than $G \sim 15$. The Reid-Hawley LF (2000) is derived from a combination of sources, including the Third Catalogue of Nearby Stars, the 8 Parsec Sample, as well as Hipparcos data. It is the local LF for the stellar disk, but it may suffer from incompleteness at magnitudes fainter than $G \sim 16$. Accordingly, our standard assumption is that the LF is flat in the magnitude range $10<M_{G}<20$ and follows the ReidHawley LF when $M_{G}<10$. In this case, the normalisation is chosen to be the maximum value of the stellar luminosity function of the Galactic disk as recorded in Table 7.3 of Reid \& Hawley (2000). The total numbers of stars in the range $10<G<20$ in the three models are given in Table 2 . The microlensing results reported in this paper are always calculated assuming our standard LF. This table tells us the rough scaling corrections that we need to convert the results to other LFs.

\subsection{Extinction}

We use the standard extinction law

$$
A\left(D_{\mathrm{s}}, \ell, b\right)= \begin{cases}0, & |b|>50^{\circ}, \\ \frac{0.165(1.192-|\tan b|)}{|\sin b|} \times & 10^{\circ}<|b|<50^{\circ}, \\ {\left[1-\exp \left(-\frac{D_{\mathrm{s}}|\sin b|}{h_{0}}\right)\right],} & 0^{\circ}<|b|<10^{\circ} .\end{cases}
$$

For $|b|>10^{\circ}$, this is the Sandage absorption law (Sandage 1972, Chen et al. 1998). The constant $h_{0}$ is the characteristic height of the extinction structures, which is $\sim 120$ pc. For $|b|<10^{\circ}$, a constant differential extinction $\gamma \sim 0.5$ (in magnitudes per kpc) is assumed. This is perhaps a little low, but we have chosen it to ensure that the total number of stars with magnitudes satisfying $10 \lesssim G \lesssim 20$ is $\sim 10^{9}$, for which there is good evidence from starcounts (Table 4.2 of Mihalas \& Binney 1981). Our extinction model is a reasonably accurate local description, but it does not include features like the molecular ring at $\sim 4 \mathrm{kpc}$. Nonetheless, it will be good enough for our study, as the sources are generally nearby.

In the Monte Carlo simulations, once the source distance, direction and absolute magnitude are chosen, then the extinction law is used to work out the apparent magnitude. We calculate the astrometric accuracy $\sigma_{\mathrm{a}}$ for a given source magnitude using the accuracy-magnitude relation for GAIA, listed in Table 1 .

\subsection{Density of Sources}

The density of sources at a distance $D_{\mathrm{s}}$ in the direction of Galactic longitude $\ell$ and latitude $b$ is

$\rho=\rho_{\mathrm{d}}+\rho_{\mathrm{b}}$.

Here, $\rho_{\mathrm{d}}$ is the density distribution of the Galactic disk and $\rho_{\mathrm{b}}$ is the density of the Galactic bulge. We use an exponential disk model with scalelength $R_{\mathrm{d}} \sim 3 \mathrm{kpc}$ and scaleheight $z_{\mathrm{h}} \sim 350$ pc (Gould, Bahcall \& Flynn 1997), namely

$\rho_{\mathrm{d}}=\rho_{0} \exp \left(-\left(R-R_{0}\right) / R_{\mathrm{d}}-|z| / z_{\mathrm{h}}\right)$,

where $R$ is Galactocentric distance, $z$ is the height above or below the Galactic plane, $R_{0}$ is the radial position of the Sun and $\rho_{0}$ is the local density of disk stars, which is taken as $0.10 M_{\odot} \mathrm{pc}^{-3}$ (Holmberg \& Flynn 2000). It is straightforward to establish that

$R^{2}=R_{0}^{2}+D_{\mathrm{s}}^{2} \cos ^{2} b-2 D_{\mathrm{s}} R_{0} \cos b \cos \ell, \quad z=D_{s} \sin b$.

We use the oblate axisymmetric bulge model provided by Kent (1992)

$\rho_{\mathrm{b}}(R, z)=3.53 K_{0}\left(\frac{s}{667 \mathrm{pc}}\right) M_{\odot} \mathrm{pc}^{-3}$,

where $s^{4}=R^{4}+(z / 0.61)^{4}$ and $K_{0}$ is a modified Bessel function. Kent's model is a reasonable fit to the Spacelab infrared data. However, it is known to be wrong in detail as the Galactic bulge is really a triaxial bar (e.g., Binney et al. 1991; Häfner et al. 2000). This means that our model like any axisymmetric model - will underestimate the photometric optical depth as compared to the observations in windows close to the Galactic Centre typically by a factor 2 or so (see e.g., Evans 1994, 1995; Binney 2000).

\subsection{Mass Function and Density of Lenses}

It is nearby objects that make the largest contribution to the astrometric lensing signal. Accordingly, in our Monte Carlo simulations, only the disk stars act as lenses. Halo populations of dark objects like Machos are not included. So, the density of lenses is the double exponential disk prescribed by eq (31). We generate the masses of the lenses using the composite mass function shown in Figure 11. Above $0.5 M_{\odot}$, we use a mass function (MF) derived from the Reid-Hawley LF via

$f(M)=\phi\left(M_{G}(M)\right) \frac{d M_{G}}{d M}$,

Here, $M_{G}(M)$ is the relationship between mass and absolute magnitude, as recorded in Kroupa, Tout \& Gilmore (1990). This ensures that the high-mass end of the MF (which gives most of the light) is compatible with the local LF. The lowmass end of the MF is not at all certain and its derivation 


\begin{tabular}{ccc}
\hline Parameter & Whole Sample & High Quality Sample \\
\hline$t_{\mathrm{ae}}($ days $)$ & $32(163)$ & $342(814)$ \\
$\theta_{\mathrm{E}}(\mathrm{mas})$ & $7.9(20.0)$ & $26.0(62.3)$ \\
$\tilde{r}_{\mathrm{E}}(\mathrm{au})$ & $0.7(1.3)$ & $0.4(0.7)$ \\
$\pi_{\mathrm{sl}}(\mathrm{mas})$ & $11.5(244)$ & $62.0(1066)$ \\
$\left|u_{0}\right|$ & $15(51)$ & $31(140)$ \\
$D_{\mathrm{l}}(\mathrm{pc})$ & $62(209)$ & $14(44)$ \\
$D_{\mathrm{s}}(\mathrm{pc})$ & $360(800)$ & $137(326)$ \\
$v\left(\mathrm{~km} \mathrm{~s}{ }^{-1}\right)$ & $135(137)$ & $132(134)$ \\
$\sigma_{\mathrm{a}}(\mu \mathrm{as})$ & $380(570)$ & $81(171)$ \\
$G$ magnitude & $\approx 18(18)$ & $\approx 15(16)$ \\
\hline
\end{tabular}

Table 3. This records the median and the mean (in brackets) values of the parameters of the simulated events for the whole sample and for the subset of high quality events (those with mass uncertainty less than $50 \%$ ).

from the LF is fraught with difficulties (e.g., D'Antona \& Mazzitelli 1994). Hence, for masses below $0.5 M_{\odot}$, we assume that the MF is flat in our standard model.

\subsection{Velocity Distributions}

We draw components of source and lens velocities from triaxial Gaussian distributions:

$$
\begin{aligned}
f\left(V_{R}, V_{z}, V_{\phi}\right) & =\frac{1}{(2 \pi)^{3 / 2} \sigma_{R} \sigma_{\phi} \sigma_{z}} \\
& \times \exp \left(-\frac{V_{R}^{2}}{2 \sigma_{R}^{2}}-\frac{\left(V_{\phi}-v_{\mathrm{m}}\right)^{2}}{2 \sigma_{\phi}^{2}}-\frac{V_{z}^{2}}{2 \sigma_{z}^{2}}\right)
\end{aligned}
$$

For the bulge sources, the random velocity is $\sigma_{R}=\sigma_{\phi}=$ $\sigma_{z}=100 \mathrm{kms}^{-1}$ about a mean of $50 \mathrm{~km} \mathrm{~s}^{-1}$ (McGinn et al. 1989). For the disk sources, the random component has $\sigma_{R}=34 \mathrm{kms}^{-1} \sigma_{\phi}=21 \mathrm{kms}^{-1} \sigma_{z}=18 \mathrm{~km} \mathrm{~s}^{-1}$ about a mean velocity $v_{\mathrm{m}}$ of $214 \mathrm{~km} \mathrm{~s}^{-1}$ (Edvardsson et al. 1993). The assumption of triaxial Gaussians with constant semiaxes seems reasonable and is often made in studies of Galactic microlensing (e.g., Kiraga \& Paczyński 1994, Kerins et al. 2001). Nonetheless, a more exact calculation should derive the spatial variation in the means and dispersions of the velocities of the stellar populations by solving the Jeans equations.

\section{MONTE CARLO SIMULATIONS}

We first pick the source position, velocity and magnitude, which provides the astrometric accuracy. This is done by drawing randomly from the probability distributions implied by eqs. (27) and (35), using the rejection method (e.g., Press et al. 1992). The astrometric optical depth (14) can be recast to provide the joint probability of drawing the lens distance and transverse velocity for a given source. We draw the mass of the lens from the mass function. Finally, we pick the impact parameter $u_{0}$ and the phase $t_{0}$ by drawing uniformly from the rectangular area in the lens plane of size $T_{\text {life }} v$ and $2 u_{\mathrm{a}} R_{\mathrm{E}}$ (illustrated in Figure 2 of Dominik \& Sahu (2000)). This provides us with a single simulated microlensing event, which has a variation of the centroid shift at least as large as $5 \sqrt{2} \sigma_{\text {a }}$ and which has a maximum during the lifetime of the GAIA mission.

\subsection{Distributions of the Events}

We create a set of 25000 such microlensing events, as a synthetic GAIA database. For each member of the sample, we compute the error in the estimated mass of the lens using eq (25), which of course depends on the uncertainties in the relative parallax and the angular Einstein radius. From this, we build up a subset of 2531 events for which the error in the inferred mass is less than $50 \%$. We refer to this as the sample of high quality events. Figures 12 and 13 show the distributions of the microlensing parameters for the entire sample and for the subset of high quality events. The medians and means of the distributions are given in Table 3. Note that the distributions have long tails caused by high impact parameter events and so we typically use medians rather than means, in accord with normal statistical practice.

There are a number of interesting things to notice. The mean source distance is $\sim 800 \mathrm{pc}$ for the whole sample, and about $140 \mathrm{pc}$ for the high quality events. The mean lens distances are still smaller. So, the astrometric microlensing signal seen by GAIA is overwhelmingly dominated by the very local stars. Of course, more distant microlensing events take place, but they can be much less commonly identified and still less commonly analysed to extract the parameters for the event.

For the whole sample, the optimum direction is towards the Galactic Centre. This is not surprising, as the source and lens density is highest in this direction. However, the high quality events are found preferentially at longitudes $\ell \sim 70^{\circ}$ and $260^{\circ}$. These events have longer durations than usual. The largest component of the transverse velocity is the circular motion about the centre of the Galaxy. So, it helps to be looking in directions in which the circular velocity is almost entirely along the line of sight. Our model of the Galaxy is axisymmetric, so the peaks in the dashed histogram of longitude are roughly symmetric about $\ell=0^{\circ}$. A similar effect can be seen in the distribution of Galactic latitude. Here, the high quality events have a broader distribution than the whole sample. A longer duration event is favoured by a somewhat higher latitude as the velocity component transverse to the line of sight is diminished.

In understanding the distribution of accuracies $\sigma_{\mathrm{a}}$, we recall that the events in the whole sample have been chosen to have at least a $5 \sqrt{2} \sigma_{\text {a }}$ variation in the centroid shift. If the source is faint, then the accuracy of the astrometry is poor and very few events can satisfy this stringent criterion. We recall from eq (13) that $\sigma_{\mathrm{a}}$ is proportional to $\theta_{\mathrm{E}}$ and varies inversely with $u^{2}$. So, the high quality sample is still more strongly biased to nearby luminous stars for which $\sigma_{\mathrm{a}}$ is small.

There are two ways to obtain a high quality event. Either the astrometric accuracy is good or the microlensing event has optimum characteristics, namely that the angular Einstein radius is large, the duration is long and the projected Einstein radius $\tilde{r}_{\mathrm{E}}$ is comparable to the size of the Earth's orbit around the Sun. All these characteristics are evident on comparing the full and the dashed histograms in Figure 13. The median duration of the high quality events is $\sim 350$ days, nearly a factor 10 times larger than the whole 

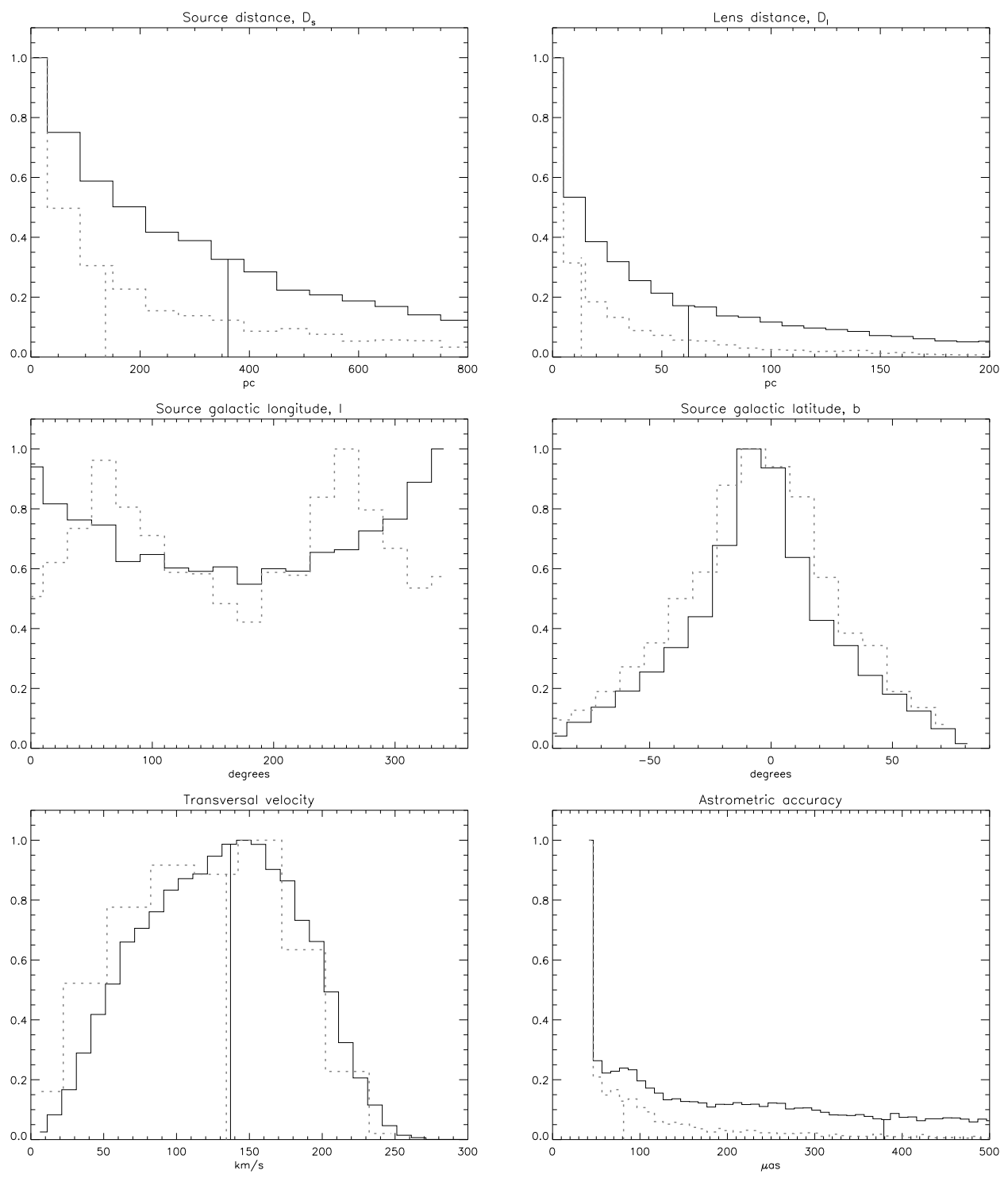

Figure 12. Distributions of source distance $D_{\mathrm{s}}$, lens distance $D_{1}$, source coordinates $(\ell, b)$, transverse velocity $v$ and astrometric accuracy $\sigma_{\mathrm{a}}$ for the simulated datasets. The histogram in full lines refers to the whole sample, dotted lines to the sub-sample of high quality events. The distributions are all normalised to a maximum value of unity. The medians are indicated by vertical bars.

sample. The projected Einstein radius $\tilde{r}_{\mathrm{E}}$ already has a median of 0.7 au for the whole sample, and it is still smaller at 0.4 au for the high quality events. The median angular Einstein radius is nearly a factor of 4 larger for the high quality events. Note that the median impact parameter for the high quality events is larger than that for the whole sample. This is because $u_{0} \sim \sqrt{\theta_{\mathrm{E}} / \sigma_{\mathrm{a}}}$. For a high quality event, it helps to have large angular Einstein radius and good accuracy, so the distribution of impact parameters has a long tail.

Table 4 shows the percentage of events for which $M, t_{\mathrm{E}}, \theta_{\mathrm{E}}$ and $\pi_{\mathrm{sl}}$ can be recovered to within $50 \%$. From the table, we see that over $40 \%$ of all the events will have good estimates for $t_{\mathrm{E}}$ and $\theta_{\mathrm{E}}$ from the GAIA astrometry alone. Current ground-based programs like MACHO and EROS attempt to recover the characteristic masses of lenses from estimates of $t_{\mathrm{E}}$ alone for samples of a few tens to hundreds of events. By contrast, GAIA will provide a much larger dataset of $\sim 10000$ microlensing events with good estimates of both $t_{\mathrm{E}}$ and $\theta_{\mathrm{E}}$. The typical locations and velocities of the sources and lenses can be inferred for the ensemble using statistical techniques based on Galactic models (in much the same manner as MACHO and EROS do at present).

The number of events with an amplification $A$ corresponding to $5 \sigma_{\mathrm{p}}$ and with at least one datapoint sampled on the photometric lightcurve by GAIA is 1260 . So, $\sim 5 \%$ of all the 25000 events have a photometric signal recorded by GAIA. One datapoint is of some help as it provides corroborating evidence that a microlensing event has taken place. If we ask for at least nine datapoints (which may very well be clustered, so there may be only three independent measurements), then the number of events is 427 . So, for only $\sim 2 \%$ of all the astrometric events will GAIA itself obtain photometry which will substantially improve the parameter estimation. 

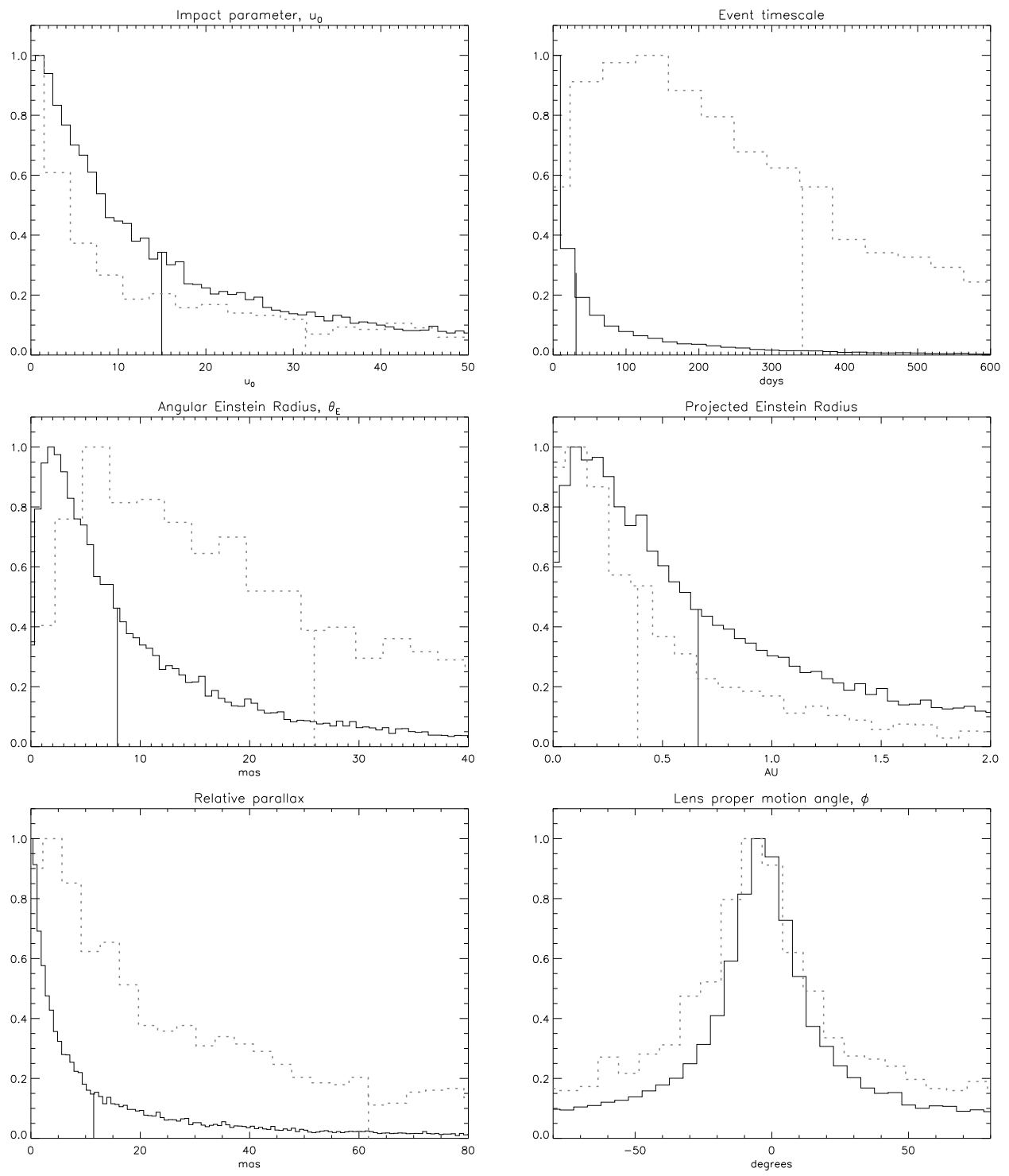

Figure 13. Distributions of impact parameter $u_{\mathrm{a}}$, event duration $t_{\mathrm{ae}}$, angular Einstein radius $\theta_{\mathrm{E}}$, projected Einstein radius $\tilde{r}_{\mathrm{E}}$, relative parallax $\pi_{\mathrm{sl}}$ and lens proper motion angle $\phi$ for the simulated datasets. The histogram in full lines refers to the whole sample, dotted lines to the sub-sample of high quality events. The distributions are all normalised to a maximum value of unity. The medians are indicated by vertical bars.

\begin{tabular}{lc}
\hline & Percentage \\
\hline$N\left(\sigma_{M}<50 \%\right)$ & $10 \%$ \\
$N\left(\sigma_{t_{\mathrm{E}}}<50 \%\right)$ & $37 \%$ \\
$N\left(\sigma_{\theta_{\mathrm{E}}}<50 \%\right)$ & $46 \%$ \\
$N\left(\sigma_{\pi_{\mathrm{s} 1}}<50 \%\right)$ & $13 \%$ \\
\hline
\end{tabular}

Table 4. This gives the percentage of events in the whole sample with an error of less than $50 \%$ in the estimate of mass $M$, or the Einstein crossing time $t_{\mathrm{E}}$, or the angular Einstein radius $\theta_{\mathrm{E}}$ or the relative parallax $\pi_{\mathrm{sl}}$.

\subsection{Determination of the Local Mass Function}

GAIA is the first instrument to have the capabilities of detecting nearby populations of very dark objects. For exam- ple, local populations of low mass $\left(\sim 0.5 M_{\odot}\right)$ black holes could easily have eluded identification thus far. Cool halo white dwarfs are also extremely difficult to detect. Existing programs look for faint objects with high proper motions characteristic of halo populations (e.g., Oppenheimer et al. 2001). Such programs miss stars with low space motions and have difficulties detecting stars with extremely high proper motions (depending on the epoch difference of the plates). So, they can only set lower limits to the local halo white dwarf density. Samples of disk white dwarfs are again selected kinematically and are subject to similar biases (Knox, Hawkins \& Hambly 1999). There may remain undetected a larger population of even fainter and cooler white dwarfs, as it is unclear whether existing surveys are probing the very faintest luminosities possible. Similarly, there may be an extensive local population of very cool brown dwarfs. The 2 Micron All Sky Survey (2MASS) and the Sloan Digital Sky 


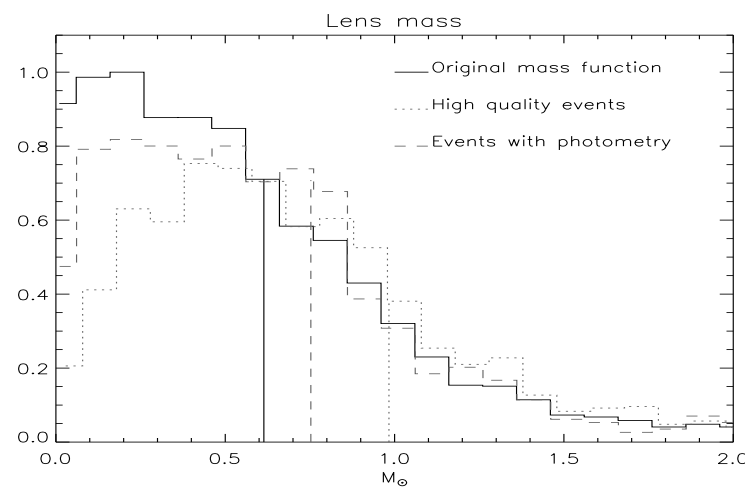

Figure 14. The distribution of masses of the lenses for the whole sample (full histogram), the subsample of high quality events (dotted histogram) and the subsample of events with some photometry (dashed histogram). The bars indicate the median of the distributions. Notice the bias at low masses in the high quality sample.

Survey (SDSS) have increased the known population of local dwarf stars with spectral types later than $\mathrm{M}$ to over a hundred (e.g., Strauss et al. 1999, Burgasser et al. 2000, Gizis, Kirkpatrick \& Wilson 2001). Nonetheless, the coolest brown dwarfs will have easily eluded the grasp of these and all current surveys. Future projects like the Space Infrared Telescope Facility (SIRTF) may be able to detect very old brown dwarfs in the solar neighbourhood by using long integration times, but a large-scale survey will be prohibitively costly in terms of time. However, the astrometric microlensing signal seen by GAIA will be sensitive to local populations of even the dimmest of stars and darkest of these objects.

Here, we investigate whether we can recover the local mass function (MF) from the astrometric microlensing signal seen by GAIA. We begin by examining the selection effects in the subsample of high quality events, the only ones for which individual lens masses can be recovered to reasonable accuracy. Figure 14 shows the distribution of masses for the entire sample and for the high quality events. The latter are biased towards masses greater than $0.3 M_{\odot}$. This is not too surprising, as it is more difficult to recover the relative parallax if the Einstein radius is small. If instead of the high quality events, we consider the subsample of events with at least one corroborating photometry datapoint, then the bias is reduced. Figure 14 already suggests that GAIA's astrometric microlensing signal will be an unbiased and powerful probe of the local population of stellar remnants, such as white dwarfs and neutron stars. However, the signal seen on smaller mass-scales will required modelling and correction for selection effects before it is unbiased.

Reid \& Hawley (2000) compute the mass function (MF) for nearby stars from the volume-limited 8 Parsec Sample using empirical mass-luminosity relationships. They obtain a power-law $\mathrm{MF}\left(f(M) \propto M^{-1}\right)$ over the range 0.1 to $1.0 M_{\odot}$. This is in rough agreement with the MF as inferred from studies of the LF of red disk stars seen with Hubble Space Telescope (Gould, Bahcall, Flynn 1997), which is a broken power-law. Above $\sim 0.6 M_{\odot}$, the MF is nearly of the classical Salpeter form with $f(M) \propto M^{-2.21}$. Below $0.6 M_{\odot}$, it is flatter than Salpeter with $f(M) \propto M^{-0.9}$ (after correction for binaries). Of course, the behaviour of the MF
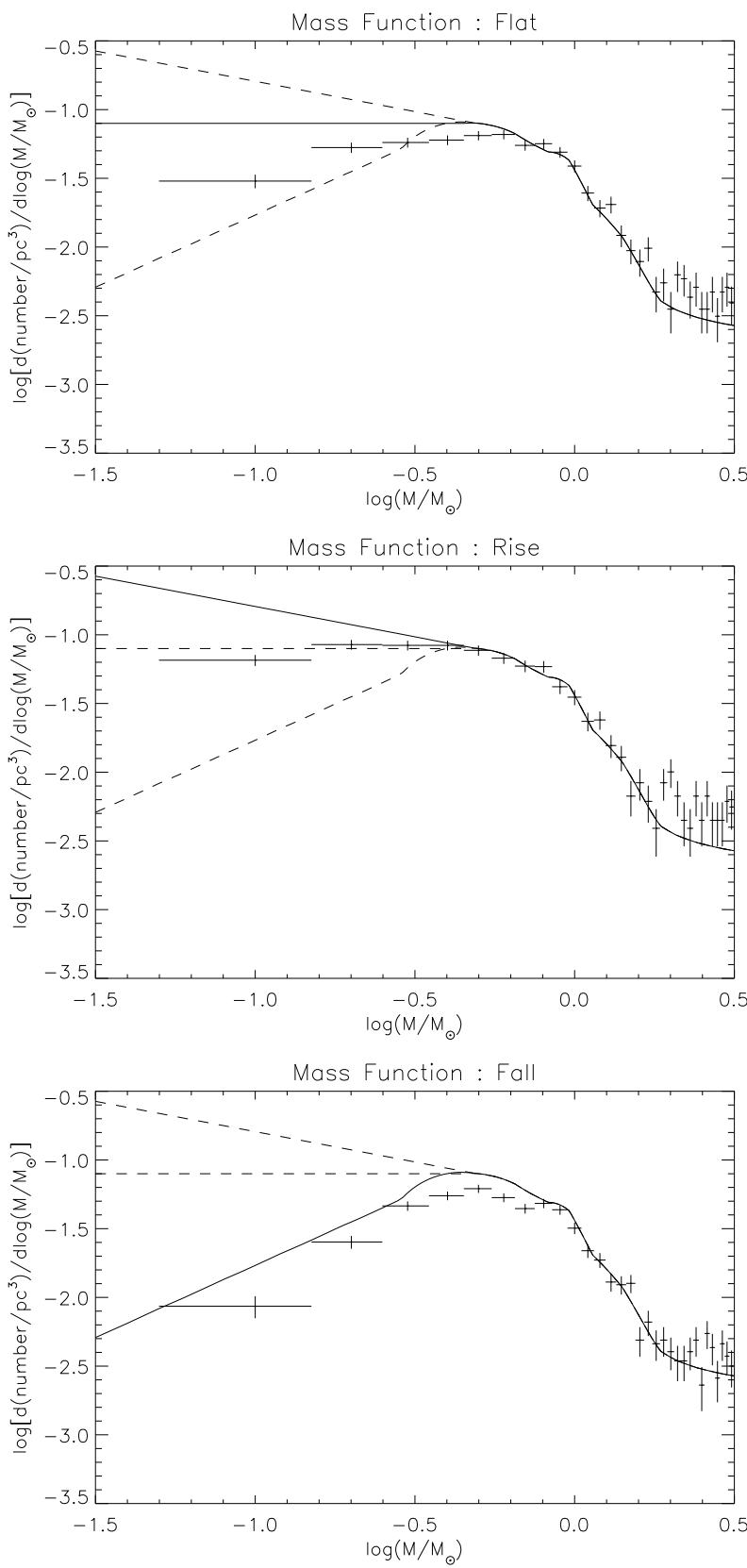

Figure 15. The recovery of the flat, rising and falling mass functions from the subsamples of high quality astrometric microlensing events generated from simulations. The vertical error bar associated with each bin is proportional to the square root of events in the bin. The horizontal error bar is the size of the bin.

becomes more uncertain as the hydrogen-burning limit is approached.

We use the three MFs shown in Figure 11 as spanning a range of reasonable possibilities. Above $0.5 M_{\odot}$, the $\mathrm{MF}$ is always derived from the Reid-Hawley luminosity function. Below $0.5 M_{\odot}$, there are three possibilities. It may be flat $\left(f(M) \propto M^{-1}\right)$, rising $\left(f(M) \propto M^{-1.44}\right)$ or falling $\left(f(M) \propto M^{0.05}\right)$. These choices are motivated by the ReidHawley LF as shown in Figure 10. For the falling MF, we choose the power-law index to be that implied by the decline in the $\mathrm{LF}$ below $M_{G} \approx 13$. For the rising $\mathrm{MF}$, we choose the 
power-law index by assuming that the rise at $M_{G} \approx 11$ continues to fainter magnitudes and that these stars are missing from the data because of incompleteness. Of course, the flat MF is so called because it has equal numbers of objects in each decade of mass and so appears flat in a plot of $\log M f(M)$ versus $\log M$. For each of the three MFs, we generate samples of 25000 astrometric microlensing events using Monte Carlo simulations. We extract the high quality events and compute the mass uncertainty using the covariance analysis. (In actual practice, the high quality events would be selected on the basis of the goodness of their $\chi^{2}$ fits). We build up the MF as a histogram. The vertical error bar associated with each bin is proportional to the square root of events in the bin. The horizontal error bar is the size of the bin.

The three cases are shown in Figure 15 with the solid line representing the underlying MF. The simulated datapoints with error bars show the MFs reconstructed from the high quality events. It is evident that GAIA can easily distinguish between the flat, rising and falling MFs. The MFs are reproduced accurately above $\sim 0.3 M_{\odot}$. Below this value, the reconstructed MFs fall below the true curves, as a consequence of the bias against smaller Einstein radii. However, this does not compromise GAIA's ability to discriminate between the three possibilities. In practice, of course, simulations could be used to re-calibrate the derived MFs at low masses and correct for the bias.

We have also carried out simulations with MFs containing spikes of compact objects, such as populations of $\sim 0.5 M_{\odot}$ white dwarfs. They lie in the mass régime to which GAIA's astrometric microlensing signal is most sensitive. So, such spikes stand out very clearly in the reconstructed MFs. We conclude that one of the major scientific contributions that GAIA can make is to determine the local MF. Microlensing provides the only way of measuring the masses of individual objects irrespective of their luminosity. GAIA is quite simply the best survey instrument to carry out an inventory of masses in the solar neighbourhood.

\section{STRATEGY}

\subsection{Detection of Events}

All sources detected by GAIA will be fit using the standard algorithms for the 6 astrometric parameters, namely $\boldsymbol{\theta}_{\mathrm{s}, 0}, \boldsymbol{\mu}_{\mathrm{s}}$ and $\mathbf{P}_{\mathrm{s}}$. This is described in, for example, volumes 1 and 3 of The Hipparcos and Tycho Catalogues (ESA 1997). So, the first cut on the dataset is to ask for large residuals in the standard astrometric parameters. Of course, microlensing is just one cause of large residuals out of many. Other possibilities include multiple companions, eclipsing systems, the mottled or spotted surfaces of stars and variability-induced movers or VIMs (Wielen 1996). VIMs were first discovered in Hipparcos data, as they give spurious negative parallaxes when fit to the standard astrometric model. In VIMs, it is hypothesised that the photometric centre of a close double star moves on the sky as one of the components has variable luminosity.

Suppose we detect the event photometrically as well as astrometrically. Then, GAIA's spectroscopy and multicolour photometry can help us distinguish microlensing events from
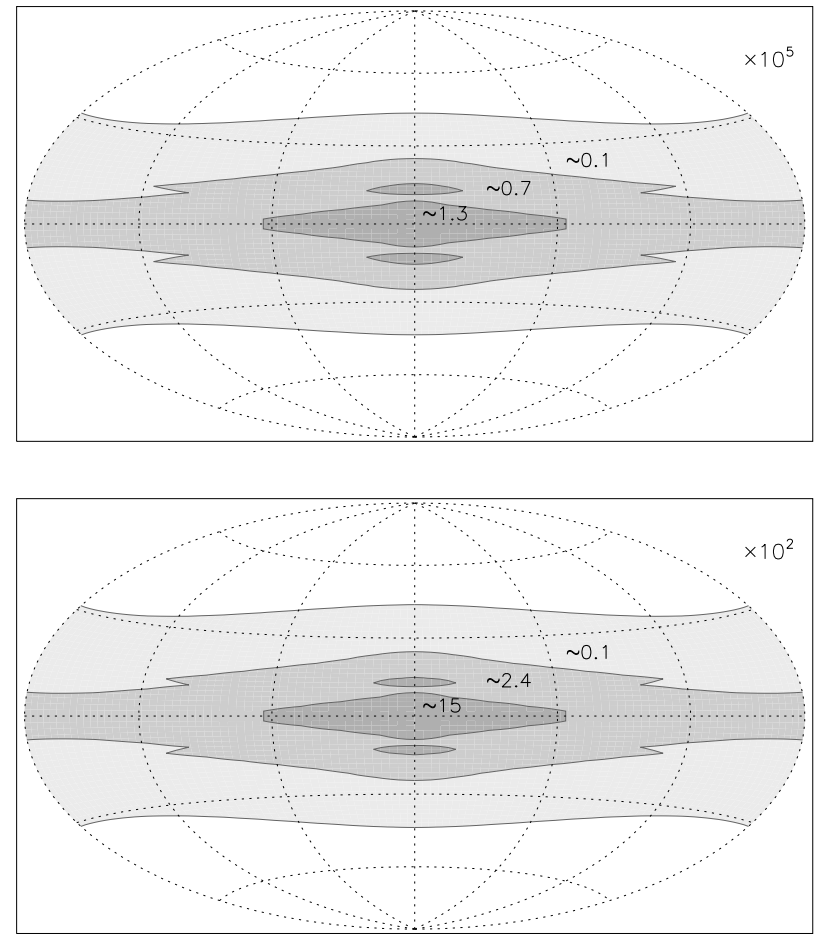

Figure 16. This shows contours of "microlensing noise" in astrometry (upper panel) and photometry (lower panel). The number within each contour refers to the total number of stars inside the shaded region enclosed between the contours that suffer an astrometric distortion or photometric brightening exceeding the mission target accuracy. The numbers are in units of $10^{5}$ in the upper panel and $10^{2}$ in the lower panel.

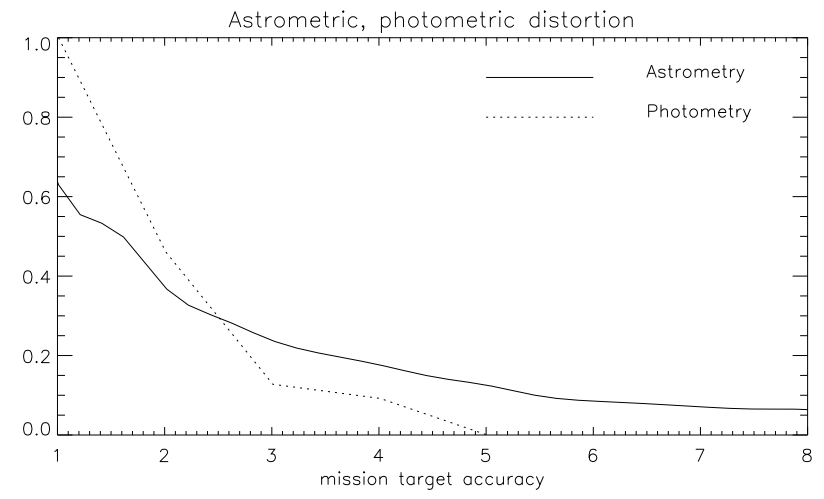

Figure 17. This shows the distribution of deviations due to microlensing at the midpoint of the mission in units of the astrometric and photometric mission target accuracy. The distributions are all normalised to a maximum value of unity. (For agiven source, the mission target accuracy is that achieved at the end of the GAIA mission. For astrometry this is better than the instantaneous accuracy listed in Table 1 by a factor of $\sim 10$ ). 
other possible causes of astrometric shifts. GAIA's spectroscopy is reasonably accurate for objects brighter than $V \sim 15$, although it covers a short wavelength range near the Ca triplet. For objects fainter than $V \sim 15$, GAIA's mulicolour photometry will be more useful. If the lens is dark, then the colours before, during and after the microlensing event are the same. Such is not true of spotted stars, VIMs and resolved binaries, which will show colour changes over the course of time. If the lens is luminous, then the colour does change over the course of the microlensing event. However, GAIA will detect such a luminous lens and so the lens proper motion and parallax will be available from a standard astrometric fit. This means that we can correct for the gravitational deflection of the source directly from the data. The multicolour photometry can also help us reduce the false detection rate. An unblended microlensing event is achromatic and does not repeat, whereas most other types of variability are chromatic and do repeat. Even long-period variables have periods of at most $\sim 600$ days (Cox 2000) and thus are short enough for GAIA to recognise as repeating.

In propitious circumstances, close companions can be distinguished from microlensing by analysis of the rate of change of the astrometric excursion with time. At times far from the closest approach, the source motion is slow in a microlensing event, whereas at or near the closest approach, the source rapidly traverses the perturbation ellipse. Although a nearby companion also causes an elliptical distortion to be superposed on the proper and parallactic motion, the traversal is harmonic in time and repeats. A close companion will also induce radial velocity variations, which may be detectable with GAIA's spectroscopy. If the stars in the binary are of similar luminosity but different colour, then there may be color variations during the centroid motion as well.

If the closest approach, which corresponds to the maximum astrometric deviation, does not occur during the lifetime of the GAIA mission, then it will be difficult to be certain that the centroid shift is caused by microlensing. This is why, in this paper, we have restricted attention only to events which satisfy the stringent criteria of a $5 \sqrt{2} \sigma_{\text {a varia- }}$ tion in the centroid shift and a maximum during the mission lifetime.

\subsection{Noise caused by Microlensing}

"Microlensing noise" is the name given to the weak effects of typically large impact parameter microlensing events. This can cause fluctuations in the positions of stars. There is some analogy with the effects of turbulence in the upper atmosphere, which causes wavefronts to become corrugated and hence causes sources to blur and twinkle. Microlensing noise is a fundamental limit to the accuracy to which we can know the positions of sources. For example, Sazhin et al. (1998, 2001) have pointed out that fluctuations of the angular positions of reference extragalactic radio and optical sources will be caused by the microlensing effects of stars in the Galaxy. Such angular fluctuations may range up to hundreds of microarcseconds. Sazhin et al. (1998) found a good example of a quasar with large positional uncertainties caused by the lensing effects of a nearby Hipparcos star; the distance of the star is very close, just 50 pcs.

Figure 16 shows a contour plot of number of sources which have an astrometric or photometric variation caused by microlensing of the same order of magnitude as the mission target accuracy. (Let us recall the mission target accuracy is that achieved at the end of the mission and so is the instantaneous astrometric accuracy given in the final line of Table 1 divided by $\sim 10$ ). The total number of sources having an astrometric deviation exceeding the mission target accuracy is $2.2 \times 10^{5}$. The positional measurement of one source in every twenty thousand is affected by microlensing noise at any instant. This number is obtained by computing $\left(2.2 \times 10^{5} / 10^{9}\right) \times\left\langle t_{\mathrm{ae}}\right\rangle / 5$ years. Many of these events will be long duration events. Any astrometric deviation will often be only slowly varying over the mission lifetime and so this will not compromise the proper motion and parallax target accuracies for GAIA. For photometry, the instantaneous number of stars affected by microlensing noise is $\sim 1.8 \times 10^{3}$. Figure 17 shows the distribution of these disturbances in units of the mission target accuracy. To be included on this Figure, an event must already have a deviation greater than the mission target accuracy. It is reassuring that the mean of the distribution of photometric deviations is strongly peaked near unity, so the blurring in photometry is mostly insignificant.

If the distribution of errors caused by instrumental effects is Gaussian, then the number of events with error less than $0.1 \sigma_{\mathrm{a}}$ is just $\sim 10 \%$. With the more realistic assumption of a non-Gaussian error distribution with higher wings the number of such deviation will be still smaller. So, if we assume that microlensing noise is a random error source, then its effects for GAIA are negligible, as in $\sim 90 \%$ of cases the microlensing noise is less than that expected from instrumental sources.

Another way in which the microlensing noise manifests itself is in distortion of the point spread function (PSF). GAIA's PSF may be approximated as a biaxial Gaussian with FWHM of 300 mas and 150 mas. To investigate the likely size of the errors, we convolve the PSF with the two micro-images corresponding to an event with Einstein ra$\operatorname{dius} \theta_{\mathrm{E}}=5$ mas. These micro-images are taken as pure $\delta$-functions. The convolved PSF is then fit with the standard biaxial Gaussian. This procedure of course carries an error, which we estimate by computing the area difference between the true PSF and fit. This is divided by the area under the PSF to give the fractional error caused by microlensing. Table 5 list the percentage error as a function of impact parameter. Although the error is small, it will be important as GAIA is aiming for a photometric accuracy of fractions of a percent.

\subsection{Photometry and Astrometry}

Figure 18 shows the uncertainty in the relative parallax as a function of astrometric accuracy for events with astrometry alone and events with astrometry and photometry. The latter curves are calculated using the covariance analysis, but now incorporating the photometric measurements as well

$b_{i j}=\sum_{k=1}^{N} \sigma_{\mathrm{a}}^{-2} \frac{\partial \theta_{\alpha}\left(t_{k}\right)}{\partial a_{i}} \frac{\partial \theta_{\alpha}\left(t_{k}\right)}{\partial a_{j}}+\sigma_{\mathrm{A}}^{-2} \frac{\partial A\left(t_{k}\right)}{\partial a_{i}} \frac{\partial A\left(t_{k}\right)}{\partial a_{j}}$,

where $A$ is the photometric amplification and

$\sigma_{\mathrm{A}}=10^{0.4 \sigma_{\mathrm{p}}}-1$. 


\begin{tabular}{ccccc}
\hline$u_{0}$ & 0.25 & 0.5 & 1 & 5 \\
$\%$ error & 0.28 & 0.26 & 0.22 & 0.14 \\
\hline
\end{tabular}

Table 5. This table lists the percentage error in the estimated point spread function against the impact parameter of the microlensing event.

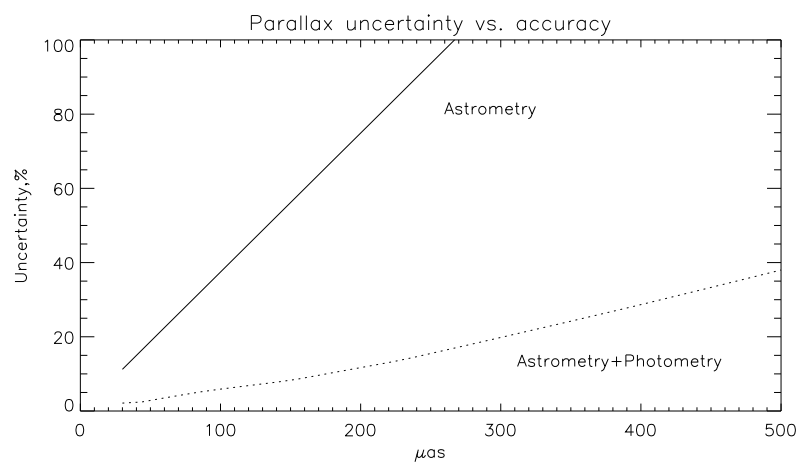

Figure 18. This shows the percentage error in the parallax of the lens as a function of the astrometric accuracy without and with additional GAIA photometry. The parameters for this event are as in Figure 1, but the impact parameter $u$ is 2.5.

The figure makes the point that just by using GAIA's photometry, it is possible to improve the accuracy of the parameter estimation by a factor of $\sim 10$. This however will only be viable for the $\sim 3 \%$ of events for which GAIA records a number of datapoints on the photometric lightcurve (see Section 5.1).

Can GAIA trigger detection of microlensing events which can subsequently be followed-up on with groundbased telescopes? This is a real challenge. GAIA provides a one-dimensional stream of astrometric data, and so must operate for roughly two years to gain enough accuracy on parallactic motions. After two years, preliminary astrometric fits may be constructed, but their accuracy will be limited. The best opportunities for detecting events will come in the latter part of the mission, when the iterative astrometric solution becomes more accurate. Hence, there is a need for at least one dedicated $1 \mathrm{~m}$ class telescope (or better still a world-wide network of such telescopes) to monitor fields in the bulge and spiral arms so that the datasets can be correlated with GAIA's database. The error is reduced by typically better than a factor of 10 when astrometric data is supplemented by densely-sampled lightcurves from the ground (see e.g., Gould \& Salim 1999).

\subsection{The FAME mission}

A related satellite is the Full-Sky Astrometric Mapping Explorer or FAME I, which is scheduled for launch in 2004 and has a mission lifetime of 2.5 years. It is based on the same principles as the Hipparcos satellite. It is less ambitious than GAIA, as it monitors mainly sources within about $2.5 \mathrm{kpc}$ of the Sun. FAME will provide astrometry on all objects with

$\dagger$ http://www.usno.navy.mil/FAME/
$6 \lesssim V \lesssim 16$. For a source with $V=10$, the accuracy of individual measurements $\sigma_{\mathrm{a}}$ is $750 \mu$ as and the mission target accuracy is $36 \mu$ as. By comparing with Table $\mathbb{1}$, we see that, roughly speaking, the accuracy of FAME's individual measurements is a factor of 10 worse than that of GAIA.

The all-sky averaged astrometric optical seen by FAME is $\sim 2 \times 10^{-6}$. There are $\sim 10^{8}$ stars with $6<V<16$, which means that FAME will measure $\sim 200$ astrometric microlensing events (with the characteristics that they have a $5 \sqrt{2} \sigma_{\text {a }}$ deviation and that the maximum occurs during the mission lifetime). This number is low, because the mission lifetime is short and the individual measurements are noisy. It is already clear that the high quality sample will be too small to deduce any useful information about the local mass function.

\section{CONCLUSIONS}

GAIA is an ambitious astrometric survey satellite that is planned for launch no later than 2012 by the European Space Agency (see ESA 2000; Perryman et al. 2001). GAIA scans the whole sky, performing multi-colour and multi-epoch photometry and spectroscopy, as well as astrometry. The reference frame is tied to a global astrometric reference frame defined by extragalactic objects. For bright sources, GAIA has the capabilities to perform individual astrometric measurements with microarcsecond accuracy. Even for objects as faint as $V \approx 20$, the positions, proper motions and parallaxes will be recovered to within at worst a few hundred microarcsecs at the end of the mission.

GAIA can measure microlensing by the small excursions of the light centroid that occur during microlensing. We use a stringent definition of an astrometric microlensing event as one with a significant variation $\left(5 \sqrt{2} \sigma_{\mathrm{a}}\right)$ of the centroid shift, together with a closest approach during the lifetime of the GAIA mission. The all-sky averaged astrometric microlensing optical depth is $\sim 2.5 \times 10^{-5}$. This means that $\sim 25000$ sources will exhibit astrometric microlensing events with such characteristics during the course of the mission. GAIA can also measure microlensing by the photometric brightening that accompanies low impact parameter events. The all-sky averaged photometric optical depth is $\sim 10^{-7}$, so there are $\sim 3600$ photometric microlensing events during the five year mission lifetime, most of which are undetectable because of the poor sampling. Consequently, very few astrometric events are measured photometrically as well. In fact, only $\sim 1260$ astrometric events have at least one datapoint sampled on the photometric lightcurve. Only for $\sim 427$ of the astrometric events will GAIA itself obtain enough photometric datapoints to improve substantially the characterisation of the event. Let us again emphasise that these numbers only refer to numbers of microlensing events with specified characteristics. Any statement as to the actual number 
of microlensing events that GAIA will find must take into account the number of false detections thrown up by the identification algorithm.

Our event statistics refer only to the signal provided by stellar lenses in the Galactic disk. We have not taken into account either stellar lenses in the Galactic bulge or dark objects in the halo (popularly called Machos). This is reasonable, as the cross-section for astrometric microlensing favours nearby lenses. The most valuable events are those for which the Einstein crossing time $t_{\mathrm{E}}$, the angular Einstein radius $\theta_{\mathrm{E}}$ and the relative parallax of the source with respect to the lens $\pi_{\mathrm{sl}}$ can all be inferred from GAIA's datastream. The mass of the lens then follows directly. If the source distance is known - for example, if GAIA itself measures the source parallax - then a complete solution of the microlensing parameters is available. Of these quantities, it is the relative parallax that is the hardest to obtain accurately. A covariance analysis is used to follow the propagation of errors and establish the conditions for recovery of the relative parallax. This happens if the angular Einstein radius $\theta_{\mathrm{E}}$ is large and the Einstein radius projected onto the observer plane $\tilde{r}_{\mathrm{E}} \sim 1$ au so that the Earth's motion about the Sun gives a substantial distortion. It is also aided if the source is bright so that GAIA's astrometric accuracy is high and if the duration of the astrometric event is long so that GAIA has time to sample it fully. These conditions favour still further lensing populations that are very close.

Monte Carlo simulations are used to establish the characteristics of the $\sim 10 \%$ of events for which GAIA can recover the mass of the lens to good accuracy. The typical lens distance is $\sim 50 \mathrm{pc}$ and the typical source distance is $\sim 300$ pc. The highest quality events seen by GAIA are overwhelmingly dominated by very local lenses.

We conclude that one of the major scientific contributions of microlensing studies with GAIA will be the determination of the mass function in the solar neighbourhood. Of course, direct mass measurements are presently possible just for binary stars with well-determined orbits. Microlensing is the only technique which can measure the masses of individual stars. GAIA is the first instrument with the ability to survey the astrometric microlensing signal provided by nearby lenses. We have used Monte Carlo simulations to show that GAIA can reconstruct the mass function in the solar neighbourhood from the sample of its highest quality events. This works particularly well for masses exceeding $\sim 0.3 M_{\odot}$. Below $0.3 M_{\odot}$, the reconstructed mass function tends to underestimate the numbers of objects, as the highest quality events are biased towards larger angular Einstein radii.

If there are local populations of low mass black holes, or very cool halo and disk white dwarfs or very old brown dwarfs, then they will have easily eluded detection with available technology. However, the astrometric microlensing signal seen by GAIA will be sensitive to local populations of even the dimmest of these stars and the darkest of these objects. GAIA is the first instrument that has the potential to map out and survey our darkest neighbours.

\section{ACKNOWLEDGMENTS}

We wish to thank Andy Gould, Shude Mao and HongSheng Zhao for a number of insightful comments, as well as Michael Perryman and Tim de Zeeuw for encouragement and information about the GAIA satellite. We are particularly indebted to Erik Høg, who provided detailed, helpful and constructive criticisms of early versions of this paper.

\section{REFERENCES}

Alcock C., et al. 1997, ApJ, 486, 697

Aubourg E., et al. 1995, AA, 301, 1

Aurière M., et al. 2001, ApJ, 553, L137

Bahcall J.N., Soneria R.M., 1980, ApJS, 44, 73

Binney J.J., Gerhard O.E., Stark A.A., Bally J., Uchida K.I. 1991, MNRAS, 252, 210

Binney J.J., 2000, In "Microlensing 2000: A New Era of Microlensing Astrophysics", eds J.W. Menzies, P.D. Sackett.

Boden A.F., Shao M., van Buren D., 1998, ApJ, 502, 538

Boutreux T., Gould A., 1996, ApJ, 462, 705

Burgasser A.J. et al. 2000, AJ, 120, 1100

Chen B., Vergely J.L., Valette B., Carraro G., 1998, A\&A, 336, 137

Cox J.P., 2000, Allen's Astrophysical Quantities, (SpringerVerlag: New York)

D'Antona F., Mazzitelli I., 1994, ApJS, 90, 467

Dominik M., Sahu K.C. 2000, ApJ, 534, 213

Edvardsson B., Andersen J., Gustafsson B., Lambert D.L., Nissen P.E., Tomkin J. 1993, A\&AS, 102, 603

ESA 1997, The Hipparcos and Tycho Catalogues, ESA SP-1200 (ESA Publications: Noordwijk)

ESA 2000, GAIA: Composition, Formation and Evolution of the Galaxy, Technical Report ESA-SCI(2000)4

Evans N.W., 1994, ApJ, 437, L31

Evans N.W., 1995, ApJ, 445, L105

Evans N.W., Kerins E.J., 2000, ApJ, 529, 917

Gizis J.E., Kirkpatrick J.D., Wilson J.C. 2001, AJ, 121, 2185

Gould A., 1995, ApJ, 441, 77

Gould A., 2000, ApJ, 542, 785

Gould A., Bahcall J.N. Flynn C., 1997, ApJ, 482, 913

Gould A., Salim S., 1999, ApJ, 524, 794

Griest K., 1991, ApJ, 366, 412

Häfner R.M., Evans N.W., Dehnen W., Binney J.J., 2000, MNRAS, 314, 433

Høg E., Novikov I.D., Polnarev A.G. 1995, A\&A, 294, 287

Holmberg J., Flynn C., 2000, MNRAS, 313, 209

Kent S.M., 1992, ApJ, 387, 181

Kerins E., Carr B.J., Evans N.W., Hewett P., Lastennet E., Le Du Y., Melchior A.-L., Smartt S., Valls-Gabaud D., 2001, MNRAS, 323, 13

Kiraga M., Paczyński B., 1994, ApJ, 430, L101

Knox R.A., Hawkins M.R.S., Hambly N.C. 1999, MNRAS, 306, 736

Kroupa P., Tout C.A., Gilmore G. 1993, 262, 545

Lindegren L., 1998, Simulation of GAIA's scanning of arbitrary directions (SAG-LL-026), unpublished

McGinn M.T., Sellgren K., Becklin E.E., Hall D.N.B. 1989, ApJ, 338,824

Miralda-Escudé J. 1996, ApJ, 470, L113

Mihalas D., Binney J., 1981, Galactic Astronomy, (Freeman: San Francisco)

Oppenheimer B.R., Hambly N.C., Digby A.C., Hodgkin S.T., Saumon D., 2001, Science, 292, 698

Paczyński B. 1996, Acta Astron, 46, 291

Perryman M.A.C., et al. 2001, AA, 369, 339 
Press W.H., Teukolsky S.A., Vetterling W.T., Flannery B.P., Numerical Recipes: The Art of Scientific Computing, (Cambridge University Press: Cambridge), chap 7.

Reid I.N., Hawley S., 2000, New Light on Dark Stars (Springer Verlag: New York), chaps 7, 8.

Sahu K.C., 1994, Nature, 370, 275

Sandage A., 1972, ApJ, 178, 1

Sazhin M.V., Zharov V.E., Volynkin A.V., Kalinina T.A. 1998, MNRAS, 300, 287

Sazhin M.V., Zharov V.E., Kalinina T.A. 2001, MNRAS, 323, 952

Strauss M.A. et al. 1999, ApJ, 522, L61

Udalski D., Szymanski M., Kaluzny J., Kubiak M., Mateo M, Krzeminski W. 1994, ApJ, 426, L69

van der Kamp P., 1967, Principles of Astrometry, (Freeman: San Francisco), p. 41.

Walker M.A. 1995, ApJ, 453, 37

Wielen R., 1996, AA, 314, 679

Zhao H.S., Evans N.W., 2000, ApJ, 545, L35 